\title{
Valence shell photoelectron angular distributions and vibrationally resolved spectra of imidazole: A combined experimental-theoretical study
}

\author{
M. Patanen, ${ }^{1}$ A. R. Abid, ${ }^{1,2}$ S. T. Pratt, ${ }^{3}$ A. Kivimäki, ${ }^{1,4}$ A. B. Trofimov, ${ }^{5,6}$ A. D. Skitnevskaya, ${ }^{5}$ \\ E. K. Grigoricheva, ${ }^{5}$ E. V. Gromov, ${ }^{5,7}$ I. Powis,${ }^{8}$ and D. M. P. Holland $\left.{ }^{9, *}\right)$
}

${ }^{1}$ Nano and Molecular Systems Research Unit, Faculty of Science, University of Oulu, P O Box 3000, 90014 Oulu, Finland

${ }^{2}$ Molecular and Condensed Matter Physics, Uppsala University, Ångströmlaboratoriet, 75237 Uppsala, Sweden

${ }^{3}$ Chemical Sciences and Engineering Division, Argonne National Laboratory, Lemont, IL 60439, USA

${ }^{4}$ MAX IV Laboratory, Lund University, P O Box 118, 22100 Lund, Sweden

${ }^{5}$ Laboratory of Quantum Chemical Modeling of Molecular Systems, Irkutsk State University, Karl Marx Str. 1, 664003 Irkutsk, Russia

${ }^{6}$ Favorsky's Institute of Chemistry, SB RAS, Favorsky Str. 1, 664033 Irkutsk, Russia

${ }^{7}$ Max-Planck Institute for Medical Research, Jahnstraße 29, 69120 Heidelberg, Germany

${ }^{8}$ School of Chemistry, The University of Nottingham, University Park, Nottingham NG7 2RD, United Kingdom

${ }^{9}$ Daresbury Laboratory, Daresbury, Warrington, Cheshire WA4 4AD, UK

\footnotetext{
*) Author to whom correspondence should be addressed: david.holland@stfc.ac.uk
} 


\begin{abstract}
Linearly polarized synchrotron radiation has been used to record polarization dependent valence shell photoelectron spectra of imidazole in the photon energy range $21-100 \mathrm{eV}$. These have allowed the photoelectron angular distributions, as characterized by the anisotropy parameter $\beta$, and the electronic state intensity branching ratios to be determined. Complementing these experimental data, theoretical photoionization partial cross sections and $\beta$-parameters have been calculated for the outer valence shell orbitals. The assignment of the structure appearing in the experimental photoelectron spectra has been guided by vertical ionization energies and spectral intensities calculated by various theoretical methods which incorporate electron correlation and orbital relaxation. Strong orbital relaxation effects have been found for the $15 \mathrm{a}^{\prime}$, nitrogen lone-pair orbital. The calculations also predict that configuration mixing leads to the formation of several low-lying satellite states. The vibrational structure associated with ionization out of a particular orbital has been simulated within the Franck-Condon model, using harmonic vibrational modes. The adiabatic approximation appears to be valid for the $X^{2} \mathrm{~A}^{\prime \prime}$ state, with the $\beta$-parameter for this state being independent of the level of vibrational excitation. However, for all the other outer valence ionic states, a disparity occurs between the observed and the simulated vibrational structure, and the measured $\beta$-parameters are at variance with the behaviour expected at the level of the Franck-Condon approximation. These inconsistencies suggest that the excited electronic states may be interacting vibronically such that the nuclear dynamics occur over coupled potential energy surfaces.
\end{abstract}




\section{INTRODUCTION}

Time-resolved, pump-probe photoelectron studies on imidazole $\left(\mathrm{C}_{3} \mathrm{H}_{4} \mathrm{~N}_{2}\right)$ have played a prominent role in the investigation of the decay of ${ }^{1} n \sigma^{*},{ }^{1} \pi \sigma^{*}$ and ${ }^{1} \pi \pi^{*}$ excited states in heteroaromatic molecules. ${ }^{1-4}$ Recently, Arbelo-González et al $^{5}$ selected imidazole to test their newly developed semiclassical method of simulating steady state and time-resolved photoelectron spectra. This modelling allowed the time dependent profile of a photoelectron band associated with a particular electronic state to be simulated. However, despite the extensive use of imidazole in time-resolved, pump-probe investigations of excited state dynamics, the steady state valence shell photoelectron spectrum remains poorly characterized, with the only published spectra ${ }^{6-8}$ being measured in the 1970s. These spectra were recorded using HeI radiation as the photon source, and hence were limited to photoelectron bands due to ionization out of the outer valence orbitals.

Imidazole has a planar $\left(\mathrm{C}_{\mathrm{s}}\right)$, five-membered heteroaromatic ring structure, and its ground state valence shell electronic configuration, at the Hartree-Fock (HF) level of theory, may be given as:

Inner valence: $\left(1 \mathrm{a}^{\prime}\right)^{2}$ through to $\left(10 \mathrm{a}^{\prime}\right)^{2}$

Outer valence: $\left(11 a^{\prime}\right)^{2}\left(12 a^{\prime}\right)^{2}\left(13 a^{\prime}\right)^{2}\left(1 a^{\prime \prime}\right)^{2}\left(14 a^{\prime}\right)^{2}\left(15 a^{\prime}\right)^{2}\left(2 a^{\prime \prime}\right)^{2}\left(3 a^{\prime \prime}\right)^{2}$

This structure is visually depicted by plots of the outer valence orbitals (Fig. 1), and a more quantitative corroborating description can be obtained from a Mulliken atomic population analysis (see Table S1, supplementary material). Imidazole's $\pi$-electron system is formed by a single $\mathrm{p}_{\mathrm{z}}$ electron from each of the $\mathrm{C}$ atoms, a single $\mathrm{p}_{z}$ electron of the $\mathrm{N}_{3}$ atom, and by the lonepair of the $\mathrm{N}_{1}$ atom. This gives rise to five $\pi$-type molecular orbitals, three of which (1a", $2 \mathrm{a}^{\prime \prime}$ and $\left.3 \mathrm{a}^{\prime \prime}\right)$ are doubly occupied in the neutral ground state. The system of $\sigma$-orbitals includes the non-bonding $15 \mathrm{a}^{\prime}$ orbital, which can be considered as a $\sigma$-type lone-pair ( $\left.\sigma_{\mathrm{N}} \mathrm{LP}\right)$ on the $\mathrm{N}_{3}$ atom. This $\sigma_{N}$ LP orbital of imidazole is analogous to the corresponding orbital of pyridine and possesses rather similar shape and localization properties. ${ }^{9,10}$ 
The aim of the present work is to study, both experimentally and theoretically, the electronic structure of the complete valence shell of imidazole, and to investigate the photoionization dynamics. Linearly polarized synchrotron radiation has been used to record high resolution, polarization dependent, photoelectron spectra of the outer valence orbitals in the photon energy range $21-100 \mathrm{eV}$. These have allowed the photoelectron angular distributions, as characterized by the anisotropy parameter $\beta$, and the electronic state intensity branching ratios (proportional to the photoionization partial cross sections) to be evaluated. Vibrational structure was observed in several of the photoelectron bands. In addition, the upper binding energy limit of the photoelectron spectrum recorded at a photon energy of $80 \mathrm{eV}$ was extended to $40 \mathrm{eV}$ to span the inner valence shell region.

The photoelectron bands appearing in the outer valence region of the experimental spectra have been assigned using vertical ionization energies calculated with the outer valence Green's function (OVGF) method, ${ }^{11-13}$ the equation-of-motion coupled cluster approach at the level of the singles and doubles model (EOM-IP-CCSD), ${ }^{14-18}$ and the linear response coupled cluster method accounting for single, double and triple excitation (CC3). ${ }^{19-22}$ A theoretical ionization spectrum of the complete valence shell was generated using the ionization energies, and the associated relative spectral intensities, obtained with the third-order algebraic-diagrammatic construction scheme $[\mathrm{ADC}(3)]$ for the one-particle Green's function. ${ }^{11,23-29}$ The ADC(3) results are applicable in regions of the spectrum where the single-electron picture of ionization breaks down, ${ }^{30}$ and are essential in the assignment of the broad photoelectron bands observed in the inner valence region of imidazole.

The photoionization dynamics of the outer valence orbitals have been investigated theoretically by employing the Continuum Multiple Scattering - X $\alpha(\mathrm{CMS}-\mathrm{X} \alpha)$ approach $^{31,32}$ to calculate photoelectron anisotropy parameters and photoionization partial cross sections.

Photoionization is often considered within the Born-Oppenheimer approximation, ${ }^{33}$ in which the electronic and nuclear motions are separated, and the nuclei move over potential energy 
surfaces formed by the electrons. Each electronic state has an associated isolated potential energy surface. Under these conditions, the vibrational structure in a specific photoelectron band may be simulated by using the Franck-Condon factors connecting the initial neutral and the final ionic states. ${ }^{34}$ Such vibrational structure will mainly consist of regular progressions involving excitation of the totally symmetric modes. Vibronic coupling, namely the interaction of two or more energetically close-lying electronic states through the nuclear motion, results in a breakdown of the Born-Oppenheimer approximation. The ensuing non-adiabatic dynamics can result in complex vibrational structure and in the excitation of non-totally symmetric vibrational modes. In the present work, the vibrational progressions in some of the outer valence photoelectron bands have been simulated in model calculations, employing the BornOppenheimer and Franck-Condon approximations, and compared to the observed structure. Differences between the predicted and measured vibrational progressions may serve as an indicator of possible vibronic interaction.

\section{EXPERIMENTAL APPARATUS AND PROCEDURE}

The photoelectron spectra of imidazole were recorded on the gas phase end station ${ }^{35}$ of the soft X-ray undulator-based FinEstBeAMS beamline ${ }^{36}$ on the $1.5 \mathrm{GeV}$ storage ring at the MAX IV Laboratory.

Synchrotron radiation for the FinEstBeAMS beamline is produced by an elliptically polarizing undulator (APPLE-II type design ${ }^{37}$ ) that allows the radiation at the experiment to be either linearly or circularly polarized. A toroidal mirror collimates the beam emitted by the undulator prior to the radiation entering a plane grating monochromator. ${ }^{38}$ The monochromator contains a 600 lines/mm grating and a 92 lines $/ \mathrm{mm}$ grating. Another toroidal mirror focusses the dispersed radiation onto the monochromator exit slit, after which an ellipsoidal mirror refocusses the monochromatic radiation into the experimental chamber. The accessible photon energy range extends from 4.5 to $1300 \mathrm{eV}$. 
The photoelectron spectra were recorded using a VG Scienta R4000 spectrometer, mounted in a fixed position, with the electron detection axis lying parallel to the plane of the electron orbit in the storage ring. The photoelectron spectra measured at photon energies between 21 and 55 $\mathrm{eV}$ were recorded using an analyser pass energy of $10 \mathrm{eV}$ and a $0.8 \mathrm{~mm}$ curved entrance slit, resulting in a theoretical spectrometer resolution of $20 \mathrm{meV}$. For photon energies between 60 and $100 \mathrm{eV}$, the analyser pass energy was increased to $20 \mathrm{eV}$, leading to a resolution of $40 \mathrm{meV}$.

The 92 lines/mm grating was used, together with a monochromator exit slit width of $100 \mu \mathrm{m}$, for the spectra recorded at photon energies up to $55 \mathrm{eV}$. The resulting theoretical optical resolution varies between $9 \mathrm{meV}$ at $21 \mathrm{eV}$ and $35 \mathrm{meV}$ at $55 \mathrm{eV}$. The 600 lines/mm grating was employed, together with a monochromator exit slit width of $130 \mu \mathrm{m}$, for the spectra recorded at higher energies. The resulting theoretical optical resolution varies between $19 \mathrm{meV}$ at $60 \mathrm{eV}$ and $41 \mathrm{meV}$ at $100 \mathrm{eV}$.

Translational Doppler broadening, associated with the thermal motion of the sample molecules, also contributes to the overall observed peak width. ${ }^{39}$ For electrons ejected with kinetic energies of $\sim 12.2$ and $91.2 \mathrm{eV}$, corresponding to the formation of the $X^{2} \mathrm{~A}^{\prime \prime}$ state in the vibrationally unexcited level at photon energies of 21 and $100 \mathrm{eV}$, the translational broadening amounts to $\sim 5.2$ and $14.3 \mathrm{meV}$, respectively.

The overall experimental resolution for the vibrationally unexcited level of the $X^{2} \mathrm{~A}^{\prime \prime}$ state, due to contributions from the electron spectrometer, the photon bandwidth, and the Doppler broadening, varied between 23 and $59 \mathrm{meV}$ at photon energies of 21 and $100 \mathrm{eV}$, respectively.

The sample of imidazole (purchased from Sigma-Aldrich with a stated purity of $>99 \%$ ) was placed in a stainless steel crucible inside a resistively heated oven. This assembly was positioned directly below the interaction region, from which the electron lens of the Scienta spectrometer accepts electrons produced by photoionization. In practice, it was found that the vapour pressure of imidazole at room temperature was sufficient to allow a good quality 
photoelectron spectrum to be recorded in a reasonable accumulation time, so that additional heating was not required.

At each photon energy, photoelectron spectra were recorded with the electric vector of the linearly polarized incident radiation lying either parallel $\left(\theta=0^{\circ}\right)$ or perpendicular $\left(\theta=90^{\circ}\right)$ to the Scienta analyser's electron acceptance axis. The orientation of the polarization could be changed by altering the settings of the undulator. Assuming electric dipole photoionization by completely linearly polarized radiation, the differential photoionization partial cross section can be expressed as: ${ }^{40}$

$\frac{\mathrm{d} \sigma}{\mathrm{d} \Omega}=\frac{\sigma}{4 \pi}\left[1+\beta \mathrm{P}_{2}(\cos \theta)\right]$

where $\sigma$ is the angle-integrated partial cross section, $\mathrm{d} \Omega$ is the differential solid angle element in the direction specified by the polar angle $\theta, \beta$ is the photoelectron anisotropy parameter, $\mathrm{P}_{2}(\cos \theta)$ is the Legendre polynomial of second order, and $\theta$ is the electron ejection angle relative to the polarization axis. $\mathrm{Eq}(1)$ can be rearranged into the more convenient form: ${ }^{41,42}$

$\beta=\frac{2\left(I_{0}-I_{90}\right)}{I_{0}+2 I_{90}}$

where $I_{0}$ and $I_{90}$ are the normalized electron intensities for parallel and perpendicular polarization orientations relative to the electron detector axis, respectively. All the spectra were normalized to the sample pressure, the accumulation time, and the photon flux prior to processing. Some of the spectra shown in this paper are so-called magic angle spectra $(\theta=$ $54.7^{\circ}$ ), where the electron intensity is independent of the photoelectron anisotropy parameter. Such magic angle spectra are synthesized from the spectra measured at $\theta=0^{\circ}$ and $\theta=90^{\circ}$ using the expression: ${ }^{41,42}$

$$
I_{\mathrm{MA}}=\frac{\left(I_{0}+2 I_{90}\right)}{3}
$$


The binding energy scale of the photoelectron spectra was calibrated using the $\mathrm{H}_{2} \mathrm{O}^{+} X^{2} \mathrm{~B}_{1}$ state ionization energy of $12.622 \mathrm{eV} .{ }^{43} \mathrm{~A}$ small signal due to water was present in most of the spectra of imidazole.

Prior to our measurements on imidazole, the performance of the electron spectrometer was checked by recording polarization dependent photoelectron spectra encompassing the Xe $5 \mathrm{p}^{5}$ ${ }^{2} \mathrm{P}_{1 / 2}$ and ${ }^{2} \mathrm{P}_{3 / 2}$ states over the same photon energy range used for our experiment on imidazole. The $\beta$-parameters derived from these spectra were in accord with the well established values. ${ }^{44-46}$ These spectra also allowed the transmission efficiency of the electron analyser as a function of electron kinetic energy to be determined, using the following procedure. The relative photoionization partial cross section of the Xe $5 p$ orbital was determined from our synthesized magic angle spectra, whilst also taking into account the incident photon flux at each photon energy. This relative Xe $5 \mathrm{p}$ photoionization partial cross section was then compared with the absolute photoionization partial cross section, ${ }^{47,48}$ thereby allowing the transmission efficiency to be deduced. All our photoelectron spectra of imidazole were normalized to the derived transmission efficiency.

The experimental photoelectron anisotropy parameter and branching ratio associated with a particular binding energy range were determined as described by Powis et al. ${ }^{41}$ Table I lists the energy ranges used to analyse the photoelectron spectra of imidazole. Within each range, a mean $\beta$-parameter is evaluated by summing the electron counts in the normalized parallel and perpendicular polarization dependent spectra and inserting these summed intensities into $\mathrm{Eq}(2)$. The intensities required for the branching ratios are evaluated in a similar manner. These mean $\beta$-parameters and branching ratios are thus vibrationally averaged values and can be compared with the corresponding theoretical predictions obtained from our fixed nuclei, CMS-X $\alpha$ calculations. Our analysis procedure also allows the variation in the $\beta$-parameter as a function of the binding energy across a specific photoelectron band to be determined. This capability enables any dependence of the $\beta$-parameter on the level of vibrational excitation to be examined. The potential errors in the $\beta$-parameters and branching ratios were estimated by 
propagation of the assumed statistical counting uncertainty through the evaluation of $\mathrm{Eq}(2)$. The resulting error bars do not include any uncertainty associated with possible systematic errors that may arise, for example, from non-ideal performance of the spectrometer or photon delivery system.

\section{COMPUTATIONAL DETAILS}

\section{A. Vertical ionization energies}

The vertical ionization energies of imidazole and the corresponding relative spectral intensities (pole strengths, $P$ ) for transitions belonging to the outer valence region were computed using several methods, including the HF theory at the level of Koopmans' theorem, the OVGF method, ${ }^{11-13}$ the third-order non-Dyson algebraic-diagrammatic construction (ADC) method $[\operatorname{IP}-\mathrm{ADC}(3)],{ }^{23-26}$ the third-order Dyson ADC method [ADC(3)], ${ }^{11,27-29}$ the equation-of-motion coupled-cluster (CC) theory for ionization potentials at the level of singles and doubles model (EOM-IP-CCSD), ${ }^{14-18}$ and the CC3 method ${ }^{19-22}$ in combination with the continuum orbital approach. ${ }^{49} \mathrm{~A}$ cc-pVTZ basis set ${ }^{50,51}$ was used in all cases (with the exception, discussed below, of the Dyson ADC(3) calculations) and the K-shell orbitals were kept frozen. Additionally, the EOM-IP-CCSD calculations were repeated using a series of the cc-pVnZ and aug-cc-pVmZ basis sets of improving quality $(n=\mathrm{D}, \mathrm{T}, \mathrm{Q}, 5 ; m=\mathrm{D}, \mathrm{T}, \mathrm{Q}),{ }^{50,51}$ thereby allowing the ionization energies to be extrapolated to the complete basis set (CBS) limit. ${ }^{52,53}$ The OVGF and CC3 calculations were performed using the Gaussian ${ }^{54}$ and CFOUR ${ }^{55}$ programs, respectively, whereas the Q-Chem ${ }^{56}$ program was employed for the IP-ADC(3) and EOM-IP-CCSD calculations.

The theoretical spectral envelope for the valence shell ionization of imidazole up to $40 \mathrm{eV}$ was generated using the results of the IP-ADC(3) and Dyson ADC(3) calculations. Both methods provide an equivalent third-order description of the ionization spectrum. Whereas IP-ADC(3) is, in general, computationally very efficient, its recent implementation ${ }^{25,26}$ still lacks a blockLanczos diagonalization procedure. Such a procedure is crucial for the generation of the spectral envelope at energies higher than those of the outer valence region where the single electron 
picture of ionization breaks down $\mathrm{n}^{30}$ and many eigenstates have to be computed. In order to recover this part of the spectrum $(\sim 23-40 \mathrm{eV})$, we resorted to the older Dyson ADC(3) scheme that allows block-Lanczos calculations to be carried out, and which has proved successful in previous studies. ${ }^{9,10,57,58}$ However, owing to the shortcomings of this older Dyson ADC(3) implementation, a smaller basis set, consisting of the cc-pVTZ basis on the second row atoms and the cc-pVDZ basis ${ }^{50,51}$ on the hydrogens, had to be used (the Cartesian representation of the $d$-functions was employed). This, however, is not expected to influence the resulting theoretical spectral profile which was constructed by convoluting the combined IPADC(3)/Dyson ADC(3) spectrum with Gaussians of $0.55 \mathrm{eV}$ FWHM (full width at half maximum). The Dyson ADC(3) calculations were performed using the original code linked to the local version of the Gamess ab initio program package..$^{59,60}$

The calculations of the vertical ionization spectra were performed using the equilibrium ground state geometrical parameters obtained by a full geometry optimization at the level of the secondorder Møller-Plesset perturbation theory (MP2) in combination with the cc-pVTZ basis sets. The Gaussian package of programs was used in the calculations. ${ }^{54}$ The computed geometrical parameters are presented in Table S2 and compared with the available experimental data.

\section{B. Franck-Condon simulations of vibrational spectra}

Franck-Condon (FC) simulations were prepared using harmonic vibrational frequencies and normal modes. The numbering of the vibrational modes follows the nomenclature recommended by Herzberg. ${ }^{61}$ The harmonic vibrational analysis required for the ground state neutral and cation used B3LYP/cc-pVTZ calculations. For the excited cation states, we used time-dependent density functional theory with the B3LYP functional (TD-B3LYP) and cc$\mathrm{pV} X \mathrm{Z}(X=\mathrm{D}, \mathrm{T})$ bases. Franck-Condon factors were then calculated using the adiabatic hessian model, including Duschinsky rotations, provided in Gaussian $16,{ }^{62}$ and convoluted with a 75 $\mathrm{cm}^{-1}$ FWHM Gaussian shaping function to generate realistic spectral profiles. 


\section{CMS-Xa calculations of photoionization properties}

Photoionization properties (cross sections, $\beta$-parameters) were calculated at the same fixed, initial geometry using a static-exchange independent electron, continuum multiple scattering model $^{31,32}$ with a $\mathrm{X} \alpha$ exchange potential $(\mathrm{CMS}-\mathrm{X} \alpha)$. Our method has been described previously ${ }^{63,64}$ and is only briefly summarized here. The neutral molecule potential is modelled as overlapping spherical regions centred on each atomic site, with the whole molecule enclosed within a spherically symmetric outer sphere that extends to infinity. Within each spherical region the exchange contribution to an effective one-electron potential is represented using the Slater $\mathrm{X} \alpha$ local density approximation. ${ }^{65}$ The wavefunctions are expressed in a symmetryadapted basis of spherical harmonic functions on each centre, with radial functions obtained by direct numerical integration within the spherical zones of the potential, and the trial potential is then iterated to self-consistency. One electron continuum functions are found by solving the scattering problem with this potential after its adaptation to ensure the correct asymptotic Coulombic behaviour for the electron-ion system. Electric dipole photoionization matrix elements and hence cross sections and $\beta$-parameters may then be calculated. The calculations were performed using the MP2/cc-pVTZ optimized geometry with atomic sphere dimensions set to 0.86 of the Norman radius. ${ }^{66}$ Spherical harmonic angular basis functions ranging up to $l_{\max }=5 ; 2 ; 1$ were used in, respectively, the outer sphere, the first row atoms, and the $\mathrm{H}$ atoms. For the continuum calculations, $l_{\max }$ cut-offs were increased to $10 ; 6 ; 4$.

\section{RESULTS AND DISCUSSION}

\section{A. Assessment of calculated binding energies}

The various calculated outer valence vertical ionization energies are listed in Table II, and compared to the experimental results obtained in the present work. A strict quantitative comparison between the theoretical and experimental results is, as usual, complicated by the fact that the measured positions of the spectral maxima are only approximately related to the vertical ionization energies, which are defined as the transitions energies at the ground state equilibrium geometry. In addition, the position of the maximum in the experimental peak may sometimes appear displaced due to superposition with other neighbouring states or hot band 
ionizations. All the theoretical methods treating electron correlation and orbital relaxation (i.e., all schemes except for the HF/Koopmans' theory) yield rather consistent ionization energies which agree with the experimental values. However, a closer inspection of the theoretical results reveals some differences that are discussed in more detail.

In the OVGF and $\mathrm{ADC}(3)$ methods, the one-hole $(1 h)$ ionization processes are treated through third order of the many-body perturbation theory (PT). The $1 h$-type states are described by these computational schemes in a similar manner, so that the errors with respect to experiment, as seen from Table II, have comparable magnitude and character.

In contrast to the OVGF approach, the $\mathrm{ADC}(3)$ schemes explicitly take into account configuration interaction between the $(1 h)$ and the two-hole-one-particle $(2 h-1 p)$ cationic states, which are treated here consistently through third and first order of PT, respectively. The states with $2 h-1 p$ and mixed $1 h / 2 h-1 p$ character can therefore be qualitatively correctly reproduced in ADC(3), whereas the OVGF method fails in such situations. As can be seen from the ADC(3) $P$ values (reflecting the $1 h$ character of the final states), the $1 h / 2 h-1 p$ configuration mixing takes place in several low-lying cationic states of imidazole. In particular, the $\left(1 \mathrm{a}^{\prime \prime}\right)^{-1}$ and $\left(13 \mathrm{a}^{\prime}\right)^{-1}$ states are affected; these possess pole strength values, $P$, of $\sim 0.5$ and $\sim 0.7$, respectively (Table II). This implies that part of their intensity is transferred to $2 h-1 p$ satellites.

The lowest of these satellites $\left({ }^{2} \mathrm{~A}^{\prime \prime}\right)$ with significant intensity $(P \approx 0.21)$ is predicted, by the present calculations (Table II), as the fourth lowest state in the spectrum at 13.8/13.9 eV (depending on the $\mathrm{ADC}(3)$ variant being used). The composition of this and the other ${ }^{2} \mathrm{~A}$ " states gaining intensity from the $1 \mathrm{a}^{\prime \prime}$ orbital is presented in Table III, where the composition is quantified in terms of the weights of the most important $1 h$ and $2 h-1 p$ configurations, as computed at the IP-ADC(3) level of theory. As can be seen, the 1a" orbital gives rise to a large number of ionization transitions, most of which can be referred to as various satellites. The largest $\left(1 \mathrm{a}^{\prime \prime}\right)^{-1}$ contribution, of $51 \%$, is to the ${ }^{2} \mathrm{~A}$ " state with a vertical ionization energy of 14.90 $\mathrm{eV}$, which can be formally considered as the "main" state. The lowest satellite state at $13.8 \mathrm{eV}$ 
can therefore be considered as a shake-down satellite, ${ }^{67}$ whereas the remaining ${ }^{2} \mathrm{~A}$ " states derived from the $1 \mathrm{a}^{\prime \prime}$ orbital, lying above the main-line, represent shake-up satellites. ${ }^{30,67}$ The situation where the innermost $\pi$ orbital is involved in extensive satellite formation while its neighbours produce only distinct main ionization lines is typical for heterocyclic molecules. ${ }^{9,68-}$ ${ }^{70}$ Such selective breakdown effects for the innermost $\pi$ orbital, in contrast to the more general picture of shake-up satellite formation, have been discussed in detail in relation to pyridine ${ }^{9}$ and the halothiophenes. ${ }^{68-70}$ As follows from the analysis of the interaction matrix elements, ${ }^{68}$ the selective breakdown phenomena ${ }^{71}$ originate from the specific localization properties of the innermost and vacant $\pi$ orbitals and, more generally, from the strong electron correlation effects associated with the $\pi$ orbital subsystem in heterocyclic molecules.

The next intense satellite $\left({ }^{2} \mathrm{~A}^{\prime}\right)$ is predicted at $15.3 \mathrm{eV}$. Further, although less intense $2 h-1 p$ states are identified that can contribute to the inner valence region at higher energy, but these are not included in Table II. As explained above, these satellite states are not recovered by the OVGF calculations.

A distinctly higher level of theory is provided by the CC3 scheme which treats the $1 h$ and $2 h$ $1 p$ states through third and second order of PT, respectively. This scheme is the well-established benchmark method. Indeed, the present CC3 results agree very well with the experimental data. For three cases in Table II, the CC3 data are missing since the calculations did not converge to the correct state. The results of the second CC scheme employed in our study, EOM-IP-CCSD, are nearly as good as those obtained using the CC3 scheme, although the EOM-IP-CCSD scheme provides only second-order PT consistency for the $1 h$-type states and first-order consistency for the $2 h-1 p$ states. ${ }^{23}$ The EOM-IP-CCSD and CC3 results disagree for the $\left(11 \mathrm{a}^{\prime}\right)^{-}$ ${ }^{1}$ state, which possesses an increased $2 h-1 p$ character, where their disparity in ionization energies amounts to $0.7 \mathrm{eV}$.

Whereas the results of the $\mathrm{CC}$ and $\mathrm{ADC}(3)$ methods appear to be comparably accurate, there is an important distinction between them concerning the position of the $\left(15 \mathrm{a}^{\prime}\right)^{-1}$ state, associated 
with the $\sigma_{N}$ LP nitrogen lone-pair orbital, in the spectrum. The ADC(3) methods predict the $\left(15 \mathrm{a}^{\prime}\right)^{-1}$ state to be the third lowest state, as also do the HF/Koopmans' and OVGF calculations, while the CC methods shift it down in energy to become the second lowest state. This situation is very similar to that for the $\sigma_{\mathrm{N}} \mathrm{LP}$ orbital in pyridine where the shift is caused by the large relaxation energy associated with the ionization out of this orbital. ${ }^{9}$ In imidazole, the relaxation mechanism is essentially the same as in pyridine and consists in a screening of the $\sigma_{\mathrm{N}} \mathrm{LP}$ hole by the $\pi-\pi^{*}$ excitations. The latter is recognized $e . g$. from the notable admixtures of $\left(\sigma_{N} L P\right)^{-1}(\pi)^{-}$ ${ }^{1} \pi^{*}$-type configurations in the expansion of the $\operatorname{ADC}(3)$ eigenvectors for the $\left(15 \mathrm{a}^{\prime}\right)^{-1}$ state.

The relaxation energy can be estimated as a difference between the results of the $\mathrm{HF} /$ Koopmans' and the HF/ $\triangle \mathrm{SCF}$ calculations, (corresponding to the frozen- and relaxeddensity HF pictures, respectively). In our HF/ $\triangle \mathrm{SCF}$ calculations, the cc-pVTZ basis set was employed and the cationic states were computed at the restricted open-shell HF (ROHF) level of theory. The resulting vertical ionization energies for the $\left(3 \mathrm{a}^{\prime \prime}\right)^{-1}$ and $\left(15 \mathrm{a}^{\prime}\right)^{-1}$ states are 7.63 and $8.95 \mathrm{eV}$, respectively, which implies relaxation shifts of 1.0 and $2.90 \mathrm{eV}$, respectively. This demonstrates that the relaxation energy for the $\sigma_{\mathrm{N}} \mathrm{LP}$ orbital in imidazole is indeed very large and even exceeds the relaxation energy for the similar orbital in pyridine $(2.73 \mathrm{eV}){ }^{9}$

Since the energy gap between the $\left(15 \mathrm{a}^{\prime}\right)^{-1}$ and $\left(2 \mathrm{a}^{\prime \prime}\right)^{-1}$ states, which are interchanged in the spectrum, is rather small ( $\leq 0.2 \mathrm{eV}$ in the correlated methods), their true order is in fact still questionable and represents a subtle issue. The existing accuracy estimates for the ADC(3) and CC methods, ${ }^{26,72-74}$ however, indicate that the predictions of the CC3 scheme are likely to be more reliable. It is also important to note that they are supported by the results of the EOM-IPCCSD method.

In order to further validate our findings, we studied the basis set dependence of the EOM-IPCCSD ionization energies, extrapolating towards the CBS limit. ${ }^{52,53}$ As can be seen from the results appearing in Table IV, the systematic increase of the basis set size up to aug-cc-pVQZ does not influence the order of the cationic states but incrementally improves all the ionization 
energies by an approximately uniform shift. The extrapolated CBS estimates differ from the ccpVTZ values presented in Table II by about $+0.2 \mathrm{eV}$. The present results indicate that the ccpVTZ basis yields reasonably converged ionization energies, although it might be advantageous to augment the basis with the diffuse functions (aug-cc-pVTZ) since this brings the results distinctly closer to the CBS limit. The evaluated CBS corrections were added to the CC3/ccpVTZ results to obtain the best theoretical estimates (BTEs) for the imidazole ionization energies (Table II). For the $D^{2} \mathrm{~A}^{\prime \prime}$ and $F^{2} \mathrm{~A}^{\prime}$ states, where the $\mathrm{CC} 3$ results are missing, the EOMIP-CCSD ionization energies were used instead.

\section{B. Assignment of the photoelectron band structure}

Fig. 2(a) presents the complete inner and outer valence region photoelectron spectrum of imidazole recorded with a photon energy $h v=80 \mathrm{eV}$, while beneath it (Fig. 2(b)) is shown, for comparison, a simulation derived from the $\mathrm{ADC}(3)$ calculated ionization energies, as discussed in section III.A. The simulation provides a very reasonable account of the experimentally observed spectral profile, including the extensive satellite structure seen above $\sim 19 \mathrm{eV}$.

A more detailed example of the outer valence region of the photoelectron spectrum, recorded with parallel and perpendicularly polarized radiation, is plotted in Fig. 3. The BTEs of the vertical binding energies (Table II), as discussed in the previous sub-section, are marked against the experimental band structure. This now informs our attempts to assign electronic structure to the photoelectron bands.

The first band displays extended vibrational progressions (Figs 3 and 4). From the first peak in the experimental spectrum, at $8.842 \mathrm{eV}$, the vibrational structure extends up to $\sim 9.7 \mathrm{eV}$, with an approximate centre of gravity at $9.1 \mathrm{eV}$. This energy is very similar to the BTE value of 9.02 $\mathrm{eV}$ for ionization out of the $3 \mathrm{a}^{\prime \prime}(\pi)$ orbital, allowing an unambiguous assignment of this band. The second band, located between $\sim 9.8$ and $11.4 \mathrm{eV}$, also exhibits vibrational structure and has a maximum at $\sim 10.4 \mathrm{eV}$ (Fig. 3). According to our theoretical results, this band can be attributed to the $15 \mathrm{a}^{\prime}(\sigma \mathrm{NLP})$ and $2 \mathrm{a}^{\prime \prime}(\pi)$ orbitals, with the BTEs for their ionization energies being 10.30 
and $10.51 \mathrm{eV}$, respectively. The mean of these two values matches the position of the observed band maximum.

Towards higher binding energy, the spectrum becomes increasingly complex, and exhibits a broad, structured feature between $\sim 13.2$ and $\sim 16 \mathrm{eV}$, with maxima at $\sim 13.8$ and $\sim 14.8 \mathrm{eV}$, and a shoulder at $\sim 15.3 \mathrm{eV}$ (Figs. 2 and 3). Our calculations indicate that the maximum at $13.8 \mathrm{eV}$ originates mainly from the $14 \mathrm{a}^{\prime}(\sigma)$ orbital (Table II and Fig. 2). However, our BTE for the ionization energy of the $14 \mathrm{a}^{\prime}(\sigma)$ orbital, $14.25 \mathrm{eV}$, is higher than the energy of the experimental maximum. A possible reason for this discrepancy is that the observed peak may originate from a superposition of several electronic/vibronic transitions. This view is supported by our ADC(3) calculations which predict an intense ${ }^{2} \mathrm{~A}^{\prime \prime} 2 h-1 p$ satellite related to the $1 \mathrm{a}^{\prime \prime}(\pi)$ orbital in this energy region (see discussion above). The second maximum, at $\sim 14.8 \mathrm{eV}$, corresponds, according to our theoretical results, to ionization out of the $1 \mathrm{a}^{\prime \prime}(\pi)$ orbital, whose BTE energy is $14.81 \mathrm{eV}$, while the shoulder at $\sim 15.3 \mathrm{eV}$ can be assigned to the $12 \mathrm{a}^{\prime}(\sigma)$ orbital with a BTE energy of $15.33 \mathrm{eV}$. The $13 \mathrm{a}^{\prime}(\sigma)$ orbital, characterized by a BTE energy of $15.09 \mathrm{eV}$, apparently also contributes to the shoulder, as also do various $2 h-1 p$ satellites, predicted here by our ADC(3) calculations (Table II and Fig. 2). The high density of states, and their complex nature, make the spectral envelope observed in the energy range $13.2-16 \mathrm{eV}$ difficult to reproduce, leading to the discrepancy between the $\mathrm{ADC}(3)$ and experimental spectral profiles (Fig. 2).

The next intense feature in the experimental photoelectron spectrum, located between 17.5 and $21 \mathrm{eV}$, contains three peaks at $\sim 17.9,19.2$ and $19.9 \mathrm{eV}$ (Figs. 2 and 3). Our results indicate that only the lowest of these peaks can be tentatively assigned to a distinct transition, namely ionization out of the $11 \mathrm{a}^{\prime}(\sigma)$ orbital with a BTE energy of $18.12 \mathrm{eV}$. The remaining higher-lying peaks are built from various satellites originating from the $11 \mathrm{a}^{\prime}(\sigma), 10 \mathrm{a}^{\prime}(\sigma)$, and $9 \mathrm{a}^{\prime}(\sigma)$ orbitals (Fig. 2). The energy of $\sim 19 \mathrm{eV}$ therefore marks the onset of a more extensive breakdown in the single particle picture of ionization. ${ }^{30}$ 
Above this $19 \mathrm{eV}$ binding energy, the $\mathrm{ADC}(3)$ calculation predicts that the spectrum will consist exclusively of various $2 h-1 p$ satellites which are related to the inner valence $8 \mathrm{a}^{\prime}(\sigma), 7 \mathrm{a}^{\prime}(\sigma)$, and $6 a^{\prime}(\sigma)$ orbitals. As can be seen from the spectrum in Fig. 2, the groups of satellites associated with the $8 \mathrm{a}^{\prime}(\sigma), 7 \mathrm{a}^{\prime}(\sigma)$, and $6 \mathrm{a}^{\prime}(\sigma)$ orbitals can be assigned to the diffuse spectral features with maxima at $23.3,26.1$ and $30.6 \mathrm{eV}$, respectively.

\section{Vibrational structure and Franck-Condon harmonic simulations}

The experimental $\left(3 \mathrm{a}^{\prime \prime}\right)^{-1} X^{2} \mathrm{~A}^{\prime \prime}$ state photoelectron band is plotted in Fig. 4, together with a $300 \mathrm{~K}$ Franck-Condon harmonic vibrational simulation. The calculated harmonic frequencies are listed in Table S3 (supplementary material). The plotting offset of the internal vibrational energy scale of the simulation has been adjusted for the best alignment between the observed and the predicted vibrational structure. An expanded view of the first peak in Fig. 4 is presented in Fig. S1 (supplementary material). The FC simulation indicates that the peak maximum accurately coincides with the position of the $0-0$ origin transition, with no skewing of the peak profile resulting from the adjacent, much less intense hot band transitions. Consequently, we identify the experimental peak maximum at $8.842 \pm 0.001 \mathrm{eV}$ with the adiabatic ionization energy.

Across the whole of this first band a very satisfactory agreement has been achieved between the experimental and simulated spectra, and this allows the vibrational structure to be assigned. The simulation indicates that the principal peaks are due to excitations involving the $\mathrm{v}^{+}$and $v_{12}{ }^{+}$modes, either alone, or in various combinations with each other. In addition, smaller contributions arise from excitation of the $v_{14^{+}}$mode, either alone or in combination with the $v_{7}{ }^{+}$ or $v_{12}{ }^{+}$modes. Fig. S2 in supplementary material shows the experimental $X{ }^{2} \mathrm{~A}^{\prime \prime}$ state photoelectron band with the main peaks marked and assigned; Table S4 lists the corresponding binding energies. Experimentally derived vibrational energies of 170 and $124 \mathrm{meV}$ have been obtained for the $v_{7}{ }^{+}$and $v_{12}{ }^{+}$modes, respectively, based upon the spacing between the peak due to the adiabatic transition and that associated with the first member in each progression. Our 
calculated energies for the $\mathrm{v}_{7}^{+}$and $v_{12}{ }^{+}$modes, after applying an appropriate harmonic scaling of $0.968,{ }^{75}$ are 170 and $125 \mathrm{meV}$, respectively.

The good agreement between the experimental and simulated spectra for the first photoelectron band indicates that the FC model, including the Born-Oppenheimer approximation invoking separable nuclear and electronic motions, appears valid for the $X^{2} \mathrm{~A}^{\prime \prime}$ state.

The second photoelectron band, lying in the binding energy range $\sim 9.8-11.4 \mathrm{eV}$, is shown in Fig. 5. Experimentally, this band exhibits vibrational structure, which appears to consist of a single progression, up to a binding energy of $\sim 10.55 \mathrm{eV}$. Although the separation between the vibrational peaks is slightly irregular, the average spacing is $\sim 108 \mathrm{meV}$. According to our BTEs (Table II and Fig. 5(a)), this band should arise from ionization out of the 15a' and 2a" orbitals, with the vertical binding energy of the $15 \mathrm{a}^{\prime}$ orbital being very slightly lower $(0.21 \mathrm{eV})$ than that of the $2 \mathrm{a}^{\prime \prime}$ orbital. However, some of the alternative calculations in Table II have the ordering exchanged. In any event, the predicted small differences between these orbital energies suggest that the associated vibrational envelopes are likely to overlap. Figs. 5(b) and 5(c) compare the experimental spectrum with the FC simulated spectra due to the $15 \mathrm{a}^{\prime}$ and $2 \mathrm{a}^{\prime \prime}$ orbitals. These simulated profiles include excitations from all thermally populated vibrational levels in the neutral molecule, while, for plotting, the underlying stick spectra have been restricted to the more intense transitions to avoid an excessively congested appearance. The principal vibrational assignments suggested by these simulations are marked in the respective figure panels.

For the $15 \mathrm{a}^{\prime}$ orbital, the predicted vibrational structure (Fig. 5(b)) is dominated by a progression in the $v_{15}{ }^{+}$mode, with a much weaker progression involving excitation of the $v_{15}{ }^{+}$mode in combination with a single quantum of the $v_{12}{ }^{+}$mode. The simulated spectrum for the $2 \mathrm{a}^{\prime \prime}$ orbital (Fig. 5(c)) predicts that the principal vibrational transitions are due to two progressions, each involving excitation of the $v_{5}{ }^{+}$mode, with one of the progressions having an additional single quantum of the $v_{10}{ }^{+}$mode. These modes are illustrated in Fig. S3 (supplementary material). 
The overall agreement between the FC simulations and experiment is at best modest. Although both simulations would seem to have the ability to rationalise the distinct coarse vibrational structure on the low binding energy side of the experimental band, discrepancies increase beyond the band maximum at $\sim 10.3 \mathrm{eV}$. While, in principle, fast dissociation can lead to increased diffuseness in photoelectron bands, the loss of the coarse $\sim 0.1 \mathrm{eV}$ spaced vibrational structure observed here would imply dissociation lifetimes of just a few fs. Without further evidence we therefore discount this mechanism as a full explanation.

Significantly, the overall width of the predicted $2 a^{\prime \prime} F C$ vibrational envelope clearly exceeds that of the second photoelectron band observed in the binding energy range $9.8-11.4 \mathrm{eV}$ (Fig. 5). Hence, even if a simple overlap of the $15 a^{\prime}$ and $2 a^{\prime \prime}$ vibrational structures with an empirically adjusted offset is anticipated, the experimental band width and loss of structure to high binding energy cannot be reproduced. In fact, confidence in these FC simulation results is further eroded because the harmonic scaling factors required to induce the apparent agreement with the experimental progression are, respectively, 1.17 and 0.9. Both of these scaling factors fall well beyond the typical B3LYP harmonic frequency scaling of $\sim 0.97$ required to correct for well documented limitations of the harmonic model. ${ }^{75}$ Hence, we conclude that, unlike the $X^{2} \mathrm{~A}^{\prime \prime}$ state photoelectron band, the harmonic FC simulations here fail to provide a fully convincing model to explain the experimental observations. Going further, we may infer the likelihood of strong vibronic interaction between the $A^{2} \mathrm{~A}^{\prime}$ and $B{ }^{2} \mathrm{~A}^{\prime \prime}$ states from this behaviour and its consequent invalidation of the underlying FC assumption of nuclear dynamics on a single adiabatic potential surface. Vibronic interactions of a similar nature have recently been investigated in cis- and trans-dichloroethene. ${ }^{76-78}$

In the third photoelectron band, the BTEs for the vertical binding energies (Table II) suggest that the main contribution to the low binding energy region should be due to the $14 \mathrm{a}^{\prime}$ orbital. In the experimental spectrum of this band (Fig. 6), weak vibrational structure is observed in the binding energy range $\sim 13.2-14.2 \mathrm{eV}$. Our FC simulation for the $14 \mathrm{a}^{\prime}$ orbital ionization appears qualitatively to replicate such structure appearing on the leading edge of the experimental band, 
and to suggest a plausible width for this band (although experimentally that is obscured by developing overlap with the next three transitions whose binding energies are indicated by the BTE values marked in Fig. 6).

According to the $\mathrm{FC}$ simulation for the $14 \mathrm{a}^{\prime}$ orbital, progressions involving various combinations of the $v_{13}{ }^{+}$and $v_{14}{ }^{+}$modes, and, to a lesser extent, the $v_{10}{ }^{+}$mode, form the major features in the simulated spectrum. The experimental spacings between the vibrational peaks are somewhat irregular, but near the beginning of the band the separation is $\sim 145 \mathrm{meV}$. The calculated harmonic vibrational energies of the $v_{13}{ }^{+}$and $\nu_{14}{ }^{+}$modes are $\sim 20 \%$ lower than this experimental value (see Fig. S3, supplementary material), and without such an unexpectedly large scaling, as applied in Fig. 6, the FC simulation provides only a qualitative model for the vibrational structure observed in the third photoelectron band. It should be borne in mind, however, that a $2 h-1 p$ satellite of ${ }^{2} \mathrm{~A}^{\prime \prime}$ symmetry, associated with the $1 \mathrm{a}^{\prime \prime}$ orbital, is predicted at $13.94 \mathrm{eV}$, and hence will influence the profile of the third photoelectron band at low binding energies.

The final photoelectron peak(s) in the binding energy range $17-21 \mathrm{eV}$ is shown in Fig. 7. Unlike the lower energy peaks, it has no clear vibrational structuring, and consequently no FC simulations have been attempted.

\section{Photoelectron branching ratios}

The photoionization dynamics for the eight outermost occupied orbitals of imidazole $\left(3 \mathrm{a}^{\prime \prime}\right.$ through to $11 \mathrm{a}^{\prime}$ ) have been studied theoretically using CMS-X $\alpha$ calculations. The dipole matrix elements obtained in these calculations can be further processed to generate partial (orbital specific) cross sections and $\beta$-parameters. The former are shown plotted in Fig. S4 (supplementary material), but these cannot be directly compared with experiment as absolute cross sections were not obtained. Relative cross sections (intensities) can, however, be compared as electronic state branching ratios. In the present case, this comparison is further 
restricted since only the first photoelectron band (associated with the $X^{2} \mathrm{~A}^{\prime \prime}$ state) is due to ionization out of a single orbital ( $\left.3 \mathrm{a}^{\prime \prime}\right)$.

The experimental branching ratio for a specific photoelectron band, or group of bands, is defined as the intensity in that band divided by the sum of the intensities in all the energetically accessible bands. Thus, at each photon energy, the experimental branching ratios sum to unity. The theoretical branching ratios are similarly defined in terms of the calculated photoionization partial cross sections, normalised by the combined total cross section. Therefore, the theoretical branching ratios must also sum to unity at a given photon energy.

We compare the experimental branching ratios for the first, second and third photoelectron bands with the corresponding summed 3a", $\left(15 a^{\prime}+2 a^{\prime \prime}\right)$, and (1a", 14a', 13a', 12a') partial channel cross section ratios. The resulting branching ratios are presented in Fig. 8, where experiment and theory are seen to be in good accord over the full photon energy range.

The theoretical branching ratios display rather more structure at the lowest photon energies than is experimentally observed. On closer inspection of the calculations, this structure can be attributed to two strong $\mathrm{ka}^{\prime}$ shape resonances, below $\sim 10 \mathrm{eV}$ electron kinetic energy, that are predicted in the $\left(a^{\prime}\right)^{-1}$ photoionization channels. These are especially obvious in the $15 \mathrm{a}^{\prime}, 14 \mathrm{a}^{\prime}$, $13 \mathrm{a}^{\prime}$ partial cross sections (see Fig. S4), but it is not unexpected for such resonances to be exaggerated in fixed geometry CMS-X $\alpha$ calculations such as those performed here. ${ }^{79}$

Despite the limitations inherent in this branching ratio comparison, the overall agreement with experiment achieved by the CMS-X $\alpha$ calculations offers some assurance that they can provide meaningful descriptions and predictions for the imidazole photoionization dynamics. In the following section we consider the behaviour of the photoelectron anisotropy parameters using these calculations. 


\section{E. Photoelectron angular distributions}

An overview of how the experimental $\beta$-parameter varies across the photoelectron bands is available for the spectrum recorded at a photon energy of $24 \mathrm{eV}$ (Fig. 3). Further examples of the magic angle, outer valence photoelectron spectra and the corresponding $\beta$-parameter curves at photon energies of 26, 30 and $50 \mathrm{eV}$ may be found in Figs. S5-S7 (supplementary material). It is clear that the measured $\beta$-parameters depend upon the specific ionic state, and the photon energy, but may also show strong variation across the profile of an individual photoelectron band.

To pursue the photon energy dependences for specific photoelectron bands, vibrationally averaged experimental $\beta$-parameters, corresponding with the binding energies specified in Table I, have been extracted. Only region 1 encompasses a photoelectron band due to ionization out of a single orbital ( $\left.3 \mathrm{a}^{\prime \prime}\right)$. The binding energy ranges of the other regions have been chosen to correlate with specific structure occurring in the experimental photoelectron spectrum (Fig. 3 ) and the electron signal within a specific region is not necessarily associated with a single orbital. This mixing may arise from the overlap of a photoelectron band corresponding to a particular orbital with that of an adjacent orbital with a similar ionization energy. The breakdown of the single particle model of ionization, ${ }^{30}$ leading to the formation of satellites, may also result in the mixing of contributions from different orbitals. For example, our ADC(3) results predict that region 4 contains, in addition to the main-line associated with the $14 \mathrm{a}^{\prime}$ orbital, a satellite whose intensity originates from the $1 \mathrm{a}^{\prime \prime}(\pi)$ orbital. In Table I we have used our $\mathrm{ADC}(3)$ calculations to predict which orbitals may contribute to ionization in a specific energy range and photoelectron band.

Fig. 9(a) shows the calculated and experimental energy-dependent $\beta$ anisotropy parameters associated with the first photoelectron band (unambiguously assigned to the single $3 \mathrm{a}^{\prime \prime}$ orbital). These are in good agreement and exhibit a photon energy dependence typical of that expected for a $\pi$-orbital, ${ }^{80-82}$ namely, a rapid rise from a low value near threshold to reach a high plateau value at an electron kinetic energy of $\sim 40 \mathrm{eV}$. 
The variation in the $\beta$-parameter with increasing vibrational excitation across the $X^{2} \mathrm{~A}^{\prime \prime}$ state photoelectron band profile is shown in Fig. 4. As can be seen, the $\beta$-value exhibits some correlations with the well delineated vibrational structure, but the values coinciding with the peaks of the vibrational structure remain essentially constant and do not depend upon the level of vibrational excitation (the weak decline in $\beta$ across the band is readily attributable to the reduction in electron kinetic energy with increasing vibrational excitation at a fixed photon energy - not to any vibrational dynamics). Such a behaviour is expected for an orbital where the photoionization can be described within the Born-Oppenheimer approximation of fully decoupled electronic and nuclear motions. This inference is consistent with the good agreement found between the simulated $\left(3 \mathrm{a}^{\prime \prime}\right)^{-1} X^{2} \mathrm{~A}^{\prime \prime}$ state vibrational structure, based upon FC factors, and the observed spectrum.

The binding energy ranges for regions 2 and 3 (Table I), which encompass the second photoelectron band (Fig. 3), have been chosen such that region 2 includes all of the observed vibrational structure and region 3 covers the featureless higher binding energy range. The calculated photon energy-dependent anisotropy parameters for the $15 \mathrm{a}^{\prime}$ and $2 \mathrm{a}^{\prime \prime}$ orbitals are plotted in Fig. 9(b), together with the experimental $\beta$-parameters for these regions. A comparison between the theoretical and experimental results shows that for region 3 the energy dependence of the $\beta$-parameter is very similar to that calculated for the $2 \mathrm{a}^{\prime \prime}$ orbital, suggesting that the energetic ordering is indeed $15 \mathrm{a}^{\prime}<2 \mathrm{a}^{\prime \prime}$, as predicted (Table II) by the CC methods. On the other hand, the data for region 2 lie midway between those calculated for the $15 \mathrm{a}^{\prime}$ and $2 \mathrm{a}^{\prime \prime}$ orbitals. A likely explanation is that the $\beta$ values for the $15 \mathrm{a}^{\prime}$ are indeed lower, but that contributions from the higher $2 \mathrm{a}^{\prime \prime}$ ionization are not fully excluded by the selected binding energies for region 2.

Figs. 3 and 5 show that the $\beta$-value across the second photoelectron band starts low near the low binding energy edge and increases towards higher binding energies. At photon energies above $26 \mathrm{eV}$, the $\beta$-values clearly reach a plateau in the second half of the band (Figs S5 -S7). The observed low $\beta$-value near the beginning of the second photoelectron band, and the higher 
value reached towards the centre of the band, already noted in the Fig. 9(b) presentation, allows us to infer that the binding energy of the $15 \mathrm{a}^{\prime}$ orbital is less than that of the $2 \mathrm{a}^{\prime \prime}$ orbital, in corroboration with our best theoretical estimates of the vertical binding energies of these two orbitals.

The third photoelectron band (Fig. 6) is attributed to ionization from three $\sigma$-type orbitals (14a', $13 \mathrm{a}^{\prime}$ and $\left.12 \mathrm{a}^{\prime}\right)$ and one $\pi$-type orbital (1a"). According to our ADC(3) results (Table II), the single particle model of ionization ${ }^{30}$ is valid for the three $\sigma$-type orbitals with each orbital possessing a well-defined main-line. However, the $1 \mathrm{a}$ " main-line pole strength is only $\sim 0.5$ and a $2 h-1 p$ shake-down satellite deriving intensity from the $1 \mathrm{a}^{\prime \prime}$ orbital is predicted near the beginning of the third band. The anisotropy across this binding energy range of $13.1-16.3 \mathrm{eV}$ has been analysed by dividing it into four regions (Table I and Fig. 3). Regions 6 and 7 comprise two broad featureless bands. In contrast, vibrational structure is observed in regions 4 and 5 . The demarcation between regions 4 and 5 does not correspond to any distinct feature in the experimental spectrum and has been chosen simply to investigate whether the photoionization dynamics differ within the binding energy range $13.1-14.3 \mathrm{eV}$.

The experimental $\beta$-parameters for regions $4-7$, and the calculated anisotropy parameters for the $14 a^{\prime}, 13 a^{\prime}, 12 a^{\prime}$ and $1 a^{\prime \prime}$ orbitals, are plotted in Fig. 10. The experimental $\beta$-parameters for regions 4 and 5 are similar, as are those for regions 6 and 7. The calculated $\beta$-values for the $12 a^{\prime}, 13 a^{\prime}$ and $14 a^{\prime}$ orbitals are similar to one another and increase fairly slowly as the photon energy increases. Such an energy dependence is characteristic of ionization out of a $\sigma$-type orbital. ${ }^{80-82}$ Contrastingly, the calculated $\beta$-value for the $1 \mathrm{a}^{\prime \prime}$ orbital is everywhere larger, and shows a more rapid rate of increase, as would be expected for a $\pi$-type orbital. ${ }^{80-82}$

While all the experimental $\beta$-values are effectively midway between limits set by the theoretical predictions, suggesting that all the sampled regions may represent some averaging of $\sigma$ - and $\pi$ type orbital ionizations, the observation of the highest $\beta$-values in region 4 could indicate a significant contribution from the $1 \mathrm{a}^{\prime \prime}$ shake-down satellite transition identified by our ADC(3) 
calculations (Table II) as occurring at $13.78 \mathrm{eV}$. This satellite would thus fall on the low energy side of the third photoelectron band (Fig. 6). No theoretical $\beta$-parameter predictions are available for this satellite, but we may assume that it would be similar to the characteristic $\pi$ type $\beta$-parameter associated with the $1 \mathrm{a}^{\prime \prime}$ main-line.

The observed variation in the $\beta$-parameter across the third photoelectron band (Fig. 6) provides an alternative view of the photoelectron anisotropy, with a clear distinction between regions 4 and 5, and regions 6 and 7. The initial rise, and subsequent fall, of $\beta$ in regions 4 and 5 suggests that the intensity near the beginning of the third band arises predominantly from the $1 \mathrm{a}$ " orbital but that contributions from $\sigma$-type orbitals increase towards higher binding energy. The contribution from the $1 \mathrm{a}^{\prime \prime}$ main-line transition is, however, somewhat harder to identify. Nevertheless, the variation in the measured $\beta$-parameter appears to support the prediction from $\operatorname{ADC}(3)$ calculations of a low energy $2 h-1 p$ satellite of $\pi$-character.

The fourth photoelectron band (Fig. 7), appearing in the binding energy range $17.3-21.0 \mathrm{eV}$, arises from the $11 \mathrm{a}^{\prime}, 10 \mathrm{a}^{\prime}$ and $9 \mathrm{a}^{\prime}$ orbitals but our calculations predict (Table II) that only the $11 \mathrm{a}^{\prime}$ orbital possesses a main-line, and even in this case the pole strength is only $\sim 0.58$. Clusters of satellites due to the $11 \mathrm{a}^{\prime}$ orbital, and especially the $10 \mathrm{a}^{\prime}$ and $9 \mathrm{a}^{\prime}$ orbitals, form a broad band in the theoretical $\operatorname{ADC}(3)$ photoelectron spectrum (Fig. 2). To analyse the experimental spectrum (Fig. 7) formed by these satellites, four binding energy ranges have been defined, and these correspond to regions $8-11$ (Table I and Fig. 3). The latter two regions correspond to bands centred at binding energies of $\sim 19.2$ and $19.9 \mathrm{eV}$, while the broad band at lower binding energy is further subdivided into two regions, 8 and 9 , in response to the weak shoulder visible at $\sim 18.4 \mathrm{eV}$ in Fig. 7. Fig. 11 shows the experimental $\beta$-parameters for regions $8-11$, and the calculated anisotropy parameters for the $11 \mathrm{a}^{\prime}$ and $10 \mathrm{a}^{\prime}$ orbitals. The experimental $\beta$-parameter for region 10 is noticeably lower than those for regions 8, 9 and 11 near threshold (Figs. 3 and 7). As the photon energy increases, the $\beta$-parameters for the four regions become similar and exhibit an energy dependence typically associated with ionization out of a $\sigma$-type orbital (see Figs. S5-S7, supplementary material). The calculated $\beta$-parameter for the $10 \mathrm{a}^{\prime}$ orbital is 
significantly lower than that for the $11 \mathrm{a}^{\prime}$ orbital near threshold, perhaps suggesting that satellite states with significant contribution from the $\left(10 \mathrm{a}^{\prime}\right)^{-1}$ configuration contribute to the intensity in region 10 .

\section{CONCLUSIONS}

We have studied the valence photoelectron spectrum of room temperature imidazole vapour at high resolution using a wide range of photon energies, extending up to $100 \mathrm{eV}$. In the outer valence region we have further examined the photoelectron angular distribution, employing pairs of spectra recorded with linearly polarized radiation oriented perpendicular and parallel to the electron detector axis to extract the anisotropy parameter, $\beta$. The experiments are complemented by theoretical calculations aiming to identify the electronic and vibrational excitations and to provide photoionization cross sections and $\beta$-parameters.

$\mathrm{ADC}(3)$ calculations have been used to estimate the vertical ionization transition energies; going beyond the independent electron $(1 h)$ model, these cover various final states with $2 h-1 p$ and mixed $1 h / 2 h-1 p$ character. Such calculations are essential for interpreting the inner valence region, but in fact identify the possibility of intense $2 h-1 p$ satellites of the $\left(1 a^{\prime \prime}\right)^{-1}$ and $\left(13 a^{\prime}\right)^{-1}$ ionizations at energies as low as 13.8 and $15.3 \mathrm{eV}$, respectively, in the outer valence region of the spectrum. Conversely, and with these exceptions, the outer valence photoelectron bands observed below a binding energy of approximately $18 \mathrm{eV}$ are confirmed as direct single electron orbital ionizations, and this region has then received the more intensive examination.

Various other theoretical methods treating electron correlation and orbital relaxation have been used to estimate vertical ionization energies. Coupled cluster calculations have been extrapolated to the complete basis limit to provide a set of best theoretical estimates, which are used to provisionally assign the structure observed in the outer valence photoelectron spectrum. Nevertheless, possible ambiguities due to the close similarity of the $15 \mathrm{a}^{\prime}$ and $2 \mathrm{a}^{\prime \prime}$ binding energies and the predicted presence of the $1 a^{\prime \prime}$ and $13 a^{\prime}$ satellites in this outer valence region remain to be settled. The intensity profile of the second band in the experimental spectra, which 
is assigned to the $\left(15 \mathrm{a}^{\prime}\right)^{-1} A^{2} \mathrm{~A}^{\prime}$ and $\left(2 \mathrm{a}^{\prime \prime}\right)^{-1} B^{2} \mathrm{~A}^{\prime \prime}$ states, is not sufficient to distinguish these two contributions. The third major structure in the spectrum is more complex with three peaks that appear to correspond to overlapping bands but is predicted to encompass four main-line transitions and two satellites according to the calculated binding energies.

The measurement of the photoelectron angular distribution provides partial corroboration and clarification of the assignments. In the second photoelectron band, low values of the anisotropy parameter, $\beta$, are recorded on the low binding energy side of the band, but these rise to a higher, sustained value by the band centre. From this, the energetic ordering $15 \mathrm{a}^{\prime}<2 \mathrm{a}^{\prime \prime}$ can be inferred, based upon general characteristics of $\sigma$ - and $\pi$-orbital angular distributions and, more specifically, on theoretically predicted $\beta$-values for these states obtained in the current study. Likewise, the $\beta$-value is significantly higher in the photoelectron band around $13.8 \mathrm{eV}$ (regions 4 and 5) than it is in the adjacent bands around 14.8 and $15.4 \mathrm{eV}$ (regions 6 and 7), again taken as indicative of a strong $\pi$-type contribution to the former that may be associated with the predicted $1 \mathrm{a}^{\prime \prime}$ satellite at $14 \mathrm{eV}$ binding energy. Nevertheless, a clear contribution of the $1 \mathrm{a}^{\prime \prime}$ main-line excitation remains undetermined by this analysis.

A central aim of this study has been to establish how far the vibronic structure deviates from a simple adiabatic model. The $X^{2} \mathrm{~A}^{\prime \prime}$ state photoelectron band exemplifies this level of isolated electronic state approximation. Its distinct vibrational peaks have spacings that are essentially (with only minor scaling) reproduced by harmonic vibrational calculation, and intensities that are in excellent agreement with the calculated harmonic Franck-Condon factors. This has facilitated a full vibrational assignment. Moreover, the $\beta$-value variation correlates closely with the vibrational features and, for a given photon excitation energy, the value of $\beta$ at the vibrational peak positions is effectively constant across the $X$ state band, confirming the decoupling of electronic and vibrational motion implicit in the Franck-Condon model.

The low binding energy region of the second photoelectron band, provisionally associated above with the $15 \mathrm{a}^{\prime}$ ionization, also displays vibrational structure, appearing as a simple 
progression superimposed on a broader base of unresolved transitions. A Franck-Condon simulation with modified harmonic frequencies semi-quantitatively reproduces the first few vibrational peaks. Correspondingly, the $2 \mathrm{a}^{\prime \prime}$ Franck-Condon simulation can be caused to match the continuation of the experimental "progression", but again only after modification of the calculated harmonic frequencies, and it fails to rationalise the loss of distinct vibrational structure observed on the high binding energy side of the band. Most tellingly, its overall vibrational envelope extends well beyond the experimental width of the second photoelectron band. It is thus concluded that this band cannot be modelled as a simple overlapping superposition of two independent vibrational progressions related to ionization out of the $15 \mathrm{a}^{\prime}$ and $2 \mathrm{a}^{\prime \prime}$ orbitals. The required up- and down-scaling of the calculated harmonic frequencies indicates that curvature of both the upper and lower adiabatic potentials may be considered in some way deficient. We therefore hypothesise a vibronic coupling between these two close lying $\left(15 a^{\prime}\right)^{-1}$ and $\left(2 a^{\prime \prime}\right)^{-1}$ states, with the nuclear dynamics occurring over the coupled potentials of both states.

This does not necessarily invalidate the interpretation of the change of $\beta$-parameter noted across the second photoelectron band. Theoretically, it can be expected that when the dynamics are formed by two interacting states, the observed asymmetry will be determined by the leading term in the state expansion. ${ }^{83}$ This has been observed, for example, in the photoelectron band system due to the $A{ }^{2} \mathrm{~B}_{2}, B{ }^{2} \mathrm{~A}_{1}$, and $C^{2} \mathrm{~A}_{2}$ states of cis-dichloroethene ${ }^{76}$ with the vibronic interaction confirmed by ab initio calculation. ${ }^{77}$

The structure observed in the photoelectron band of imidazole around $14 \mathrm{eV}$ can be qualitatively reproduced by Franck-Condon simulation for the 14a' ionization (albeit with, again, a surprisingly large rescaling of the calculated harmonic frequencies) but this rather contradicts the $\beta$-parameter measurement for this band which has been interpreted as indicating a significant $\pi$-type character, putatively from the $1 \mathrm{a}^{\prime \prime}$ satellite. In fact, however, the $\beta$-parameter varies strongly across the band, which might result from either the blending together of two or more overlapping, non-interacting state ionizations or from $\beta$ displaying a non-Franck-Condon 
dependence on the degree of vibrational excitation. This again raises the possibility of vibronic coupling between at least some of the adjacent ionic states $\left(C^{2} \mathrm{~A}^{\prime}, D^{2} \mathrm{~A}^{\prime \prime}, E^{2} \mathrm{~A}^{\prime \prime}\right.$ and $\left.F^{2} \mathrm{~A}^{\prime}\right)$ associated with the third photoelectron band.

Recent studies on cis- and trans-dichloroethene ${ }^{76-78}$ have investigated the influence of nonadiabatic nuclear dynamics in the outer valence ionic states. In particular, the theoretical modelling predicted vibronic interactions between the $A^{2} \mathrm{~B}_{2}, B^{2} \mathrm{~A}_{1}$, and $C^{2} \mathrm{~A}_{2}$ states, and the $D$ ${ }^{2} \mathrm{~B}_{1}$ and $E{ }^{2} \mathrm{~B}_{2}$ states, leading to complex photoelectron band systems. Moreover, the experimental anisotropy parameters, measured across a particular band system, exhibited a highly irregular dependence upon the level of vibrational excitation. Such an energy dependence is at odds with expectations based upon the adiabatic approximation of isolated electronic states, each having an associated (non-interacting) potential energy surface. The similarity between some of the present results for imidazole, and those for dichloroethene, suggest that vibronic coupling may be affecting the valence ionic states of imidazole. An ab initio investigation of the possible vibronic coupling in imidazole is ongoing.

\section{SUPPLEMENTARY MATERIAL}

See the supplementary material for the presentation of additional experimental spectra, tables of computed geometry and vibrational modes, and a table giving cation vibrational energies.

\section{ACKNOWLEDGMENTS}

D.M.P.H is grateful to the Science and Technology Facilities Council (United Kingdom) for financial support. S.T.P is supported by the U.S. Department of Energy, Office of Science, Office of Basic Energy Sciences, Division of Chemical Sciences, Geosciences, and Biosciences under contract No. DE-AC02-06CH11357. A.B.T., E.V.G., E.K.G., and A.D.S gratefully acknowledge the grant, Grant No. FZZE-2020-0025, from the Ministry of science and higher education of the Russian Federation. A.D.S. also gratefully acknowledges the Russian Foundation for Basic Research according to the research project No. 19-03-00947. M.P. 
acknowledges the Academy of Finland for financial support. A.R.A acknowledges the Horizon 2020 programme I4Future for financial support (Grant agreement no. 713606). The University of Nottingham High Performance Computing Facility provided computational resources partially supporting this investigation. We acknowledge MAX IV Laboratory for time on Beamline FinEstBeAMS under Proposal 20190182. Research conducted at MAX IV, a Swedish national user facility, is supported by the Swedish Research council under contract 2018-07152, the Swedish Governmental Agency for Innovation Systems under contract 2018-04969, and Formas under contract 2019-02496. We thank the technical staff for their support and for the smooth operation of the facility.

\section{DATA AVAILABILITY}

Raw data were generated at the MAXIV synchrotron radiation facility. Derived data supporting the findings of this study are available from the corresponding author upon reasonable request. 


\section{References}

[1] M. N. R. Ashfold, B. Cronin, A. L. Devine, R. N. Dixon, and M. G. D. Nix, Science, 312, 1637 (2006).

[2] A. L. Devine, B. Cronin, M. G. D. Nix, and M. N. R. Ashfold, J. Chem. Phys. 125, $184302(2006)$.

[3] D. J. Hadden, K. L. Wells, G. M. Roberts, L. T. Bergendahl, M. J. Paterson, and V. G. Stavros, Phys. Chem. Chem. Phys. 13, 10342 (2011).

[4] H. Yu, N. L. Evans, V. G. Stavros, and S. Ullrich, Phys. Chem. Chem. Phys. 14, 6266 (2012).

[5] W. Arbelo-González, R. Crespo-Otero, and M. Barbatti, J. Chem. Theory Comput. 12, 5037 (2016).

[6] S. Cradock, R. H. Findlay, and M. H. Palmer, Tetrahedron, 29, 2173 (1973).

[7] L. Klasinc, B. Ruščić, F. Kajfež, and V. Šunjić, Int. J. Quant. Chem. Quant. Biol. Symp. 5, 367 (1978).

[8] B. G. Ramsey, J. Org. Chem. 44, 2093 (1979).

[9] A. B. Trofimov, D. M. P. Holland, I. Powis, R. C. Menzies, A. W. Potts, L. Karlsson, E. V. Gromov, I. L. Badsyuk, and J. Schirmer, J. Chem. Phys. 146, 244307 (2017).

[10] D. M. P. Holland, I. Powis, A. B. Trofimov, R. C. Menzies, A. W. Potts, L. Karlsson, I. L. Badsyuk, T. E. Moskovskaya, E. V. Gromov, and J. Schirmer, J. Chem. Phys. 147, 164307 (2017).

[11] W. von Niessen, J. Schirmer, and L. S. Cederbaum, Comp. Phys. Reports, 1, 57 (1984).

[12] V. G. Zakrzewski and W. von Niessen, J. Comput. Chem. 14, 13 (1993).

[13] V. G. Zakrzewski and J. V. Ortiz, Int. J. Quantum Chem. 52, 23 (1994).

[14] H. Sekino and R. J. Bartlett, Int. J. Quantum Chem. 26, 255 (1984).

[15] J. Geertsen, M. Rittby, and R. J. Bartlett, Chem. Phys. Lett. 164, 57 (1995).

[16] J. F. Stanton and J. Gauss, J. Chem. Phys. 103, 1064 (1995).

[17] M. Nooijen and J. G. Snijders, J. Chem. Phys. 102, 1681 (1995).

[18] A. I. Krylov, Annu. Rev. Phys. Chem. 59, 433 (2008). 
[19] O. Christiansen, H. Koch, and P. Jørgensen, J. Chem. Phys. 103, 7429 1995).

[20] H. Koch, H. J. A. Jensen, P. Jørgensen, and T. Helgaker, J. Chem. Phys. 93, 3345 (1990).

[21] H. Koch and P. Jørgensen, J. Chem. Phys. 93, 3333 (1990).

[22] E. Dalgaard and H. J. Monkhorst, Phys. Rev. A 28, 1217 (1983).

[23] J. Schirmer, A. B. Trofimov, and G. Stelter, J. Chem. Phys. 109, 4734 (1998).

[24] A. B. Trofimov and J. Schirmer, J. Chem. Phys. 123, 144115 (2005).

[25] A. L. Dempwolff, A. C. Paul, A. M. Belogolova, A. B. Trofimov, and A. Dreuw, J. Chem. Phys. 152, 024113 (2020).

[26] A. L. Dempwolff, A. C. Paul, A. M. Belogolova, A. B. Trofimov, and A. Dreuw, J. Chem. Phys. 152, 024125 (2020).

[27] J. Schirmer, L. S. Cederbaum, and O. Walter, Phys. Rev. A 281237 (1983).

[28] J. Schirmer and G. Angonoa, J. Chem. Phys. 91, 1754 (1989).

[29] H. -G. Weikert, H. -D. Meyer, L. S. Cederbaum, and F. Tarantelli, J. Chem. Phys. 104, 7122 (1996).

[30] L. S. Cederbaum, W. Domcke, J. Schirmer, and W. von Niessen, Adv. Chem. Phys. 65, 115 (1986).

[31] D. Dill and J. L. Dehmer, J. Chem. Phys. 61, 692 (1974).

[32] J. W. Davenport, Ph.D. thesis (University of Pennsylvania, 1976).

[33] M. Born and R. Oppenheimer, Ann. Phys. 389, 457 (1927).

[34] G. Herzberg, Molecular Spectra and Molecular Structure III: Electronic Spectra and Electronic Structure of Polyatomic Molecules (Van Nostrand Reinhold, New York, 1966).

[35] K. Kooser, A. Kivimäki, P. Turunen, R. Pärna, L. Reisberg, M. Kirm, M. Valden, M. Huttula, and E. Kukk, J. Synchrotron Rad. 27, 1080 (2020).

[36] R. Pärna, R. Sankari, E. Kukk, E. Nõmmiste, M. Valden, M. Lastusaari, K. Kooser, K. Kokko, M. Hirsimäki, S. Urpelainen, P. Turunen, A. Kivimäki, V. Pankratov, L. Reisberg, F. Hennies, H. Tarawneh, R. Nyholm, and M. Huttula, Nucl. Instrum. Methods Phys. Res. A 859, 83 (2017). 
[37] S. Sasaki, K. Kakuno, T. Takada, T. Shimada, K. Yanagida, and Y. Miyahara, Nucl. Instrum. Methods Phys. Res. A 331, 763 (1993).

[38] R. Follath and F. Senf, Nucl. Instrum Methods Phys. Res. A 390, 388 (1997).

[39] P. Baltzer, L. Karlsson, M. Lundqvist, and B. Wannberg, Rev. Sci. Instrum. 64, 2179 (1993).

[40] J. Cooper and R. Zare, J. Chem. Phys. 48, 942 (1968).

[41] I. Powis, D. M. P. Holland, E. Antonsson, C. Nicolas, C. Miron, M. Schneider, D. Yu. Soshnikov, A. Dreuw, and A. B. Trofimov, J. Chem. Phys. 143, 144304 (2015).

[42] D. M. P. Holland, S. Nandi, C. Nicolas, J. D. Bozek, M. Patanen, and I. Powis, Chem. Phys. 542, 111050 (2021).

[43] J. E. Reutt, L. S. Wang, Y. T. Lee, and D. A. Shirley, J. Chem. Phys. 85, 6928 (1986).

[44] D. M. P. Holland, A. C. Parr, D. L. Ederer, J. L. Dehmer, and J. B. West, Nucl, Instrum. Methods Phys. Res. 195, 331 (1982).

[45] S. H. Southworth, A. C. Parr, J. E. Hardis, J. L. Dehmer, and D. M. P. Holland, Nucl. Instrum. Methods Phys. Res. A 246, 782 (1986).

[46] M. O. Krause, T. A. Carlson, and P. R. Woodruff, Phys. Rev. A 24, 1374 (1981).

[47] A. Fahlman, M. O. Krause, T. A. Carlson, and A. Svensson, Phys. Rev, A 30, 812 (1984).

[48] U. Becker, D. Szostak, H. G. Kerkhoff, M. Kupsch, B. Langer, R. Wehlitz, A. Yagishta, and T. Hayaishi, Phys. Rev. A 39, 3902 (1989).

[49] J. F. Stanton and J. Gauss, J. Chem. Phys. 111, 8785 (1999).

[50] T. H. Dunning, J. Chem. Phys. 90, 1007 (1989).

[51] R. A. Kendall, T. H. Dunning, and R. J. Harrison, J. Chem. Phys. 96, 6796 (1992).

[52] D. Feller, J. Chem. Phys. 96, 6104 (1992).

[53] D. Feller, J. Chem. Phys. 98, 7059 (1993).

[54] M. J. Frisch, G. W. Trucks, H. B. Schlegel, G. E. Scuseria, M. A. Robb, J. R. Cheeseman, G. Scalmani, V. Barone, B. Mennucci, G. A. Petersson, H. Nakatsuji, M. Caricato, X. Li, H. P. Hratchian, A. F. Izmaylov, J. Bloino, G. Zheng, J. L. Sonnenberg, M. Hada, M. Ehara, K. Toyota, R. Fukuda, J. Hasegawa, M. Ishida, T. Nakajima, Y. 
Honda, O. Kitao, H. Nakai, T. Vreven, J. A. Montgomery, Jr., J. E. Peralta, F. Ogliaro, M. Bearpark, J. J. Heyd, E. Brothers, K. N. Kudin, V. N. Staroverov, T. Keith, R. Kobayashi, J. Normand, K. Raghavachari, A. Rendell, J. C. Burant, S. S. Iyengar, J. Tomasi, M. Cossi, N. Rega, J. M. Millam, M. Klene, J. E. Knox, J. B. Cross, V. Bakken, C. Adamo, J. Jaramillo, R. Gomperts, R. E. Stratmann, O. Yazyev, A. J. Austin, R. Cammi, C. Pomelli, J. W. Ochterski, R. L. Martin, K. Morokuma, V. G. Zakrzewski, G. A. Voth, P. Salvador, J. J. Dannenberg, S. Dapprich, A. D. Daniels, O. Farkas, J. B. Foresman, J. V. Ortiz, J. Cioslowski, and D. J. Fox, Gaussian 09, Revision C.01, Gaussian, Inc., Wallingford, CT, 2010.

CFOUR, Coupled cluster techniques for Computational Chemistry, a quantumchemical program package by J. F. Stanton, J. Gauss, M. E. Harding, and P. G. Szalay, with contributions from A. A. Auer, R. J. Bartlett, U. Benedikt, C. Berger, D. E. Bernholdt, Y. J. Bomble, L. Cheng, O. Christiansen, M. Heckert, O. Heun, C. Huber, T.-C. Jagau, D. Jonsson, J. Jusélius, K. Klein, W. J. Lauderdale, D. A. Matthews, T. Metzroth, L. A. Mück, D. P. O’Neill, D. R. Price, E. Prochnow, C. Puzzarini, K. Ruud, F. Schiffmann, W. Schwalbach, C. Simmons, S. Stopkowicz, A. Tajti, J. Vázquez, F. Wang, and J. D. Watts and the integral packages MOLECULE (J. Almlöf and P. R. Taylor), PROPS (P. R. Taylor), ABACUS (T. Helgaker, H. J. Aa. Jensen, P. Jørgensen, and J. Olsen), and ECP routines by A. V. Mitin and C. van Wüllen. For the current version, see http://www.cfour.de. Y. Shao, Z. Gan, E. Epifanovsky, A. T. B. Gilbert, M. Wormit, J. Kussmann, A. W. Lange, A. Behn, J. Deng, X. Feng, D. Ghosh, M. Goldey, P. R. Horn, L. D. Jacobson, I. Kaliman, R. Z. Khaliullin, T. Kuś, A. Landau, J. Liu, E. I. Proynov, Y. M. Rhee, R. M. Richard, M. A. Rohrdanz, R. P. Steele, E. J. Sundstrom, H. L. Woodcock, P. M. Zimmerman, D. Zuev, B. Albrecht, E. Alguire, B. Austin, G. J. O. Beran, Y. A. Bernard, E. Berquist, K. Brandhorst, K. B. Bravaya, S. T. Brown, D. Casanova, C.-M. Chang, Y. Chen, S. H. Chien, K. D. Closser, D. L. Crittenden, M. Diedenhofen, R. A. DiStasio, H. Do, A. D. Dutoi, R. G. Edgar, S. Fatehi, L. Fusti-Molnar, A. Ghysels, A. GolubevaZadorozhnaya, J. Gomes, M. W. D. Hanson-Heine, P. H. P. Harbach, A. W. Hauser, E. 
G. Hohenstein, Z. C. Holden, T.-C. Jagau, H. Ji, B. Kaduk, K. Khistyaev, J. Kim, J. Kim, R. A. King, P. Klunzinger, D. Kosenkov, T. Kowalczyk, C. M. Krauter, K. U. Lao, A. D. Laurent, K. V. Lawler, S. V. Levchenko, C. Y. Lin, F. Liu, E. Livshits, R. C. Lochan, A. Luenser, P. Manohar, S. F. Manzer, S.-P. Mao, N. Mardirossian, A. V. Marenich, S. A. Maurer, N. J. Mayhall, E. Neuscamman, C. M. Oana, R. OlivaresAmaya, D. P. O’Neill, J. A. Parkhill, T. M. Perrine, R. Peverati, A. Prociuk, D. R. Rehn, E. Rosta, N. J. Russ, S. M. Sharada, S. Sharma, D. W. Small, A. Sodt, T. Stein, D. Stück, Y.-C. Su, A. J. W. Thom, T. Tsuchimochi, V. Vanovschi, L. Vogt, O. Vydrov, T. Wang, M. A. Watson, J. Wenzel, A. White, C. F. Williams, J. Yang, S. Yeganeh, S. R. Yost, Z.-Q. You, I. Y. Zhang, X. Zhang, Y. Zhao, B. R. Brooks, G. K. L. Chan, D. M. Chipman, C. J. Cramer, W. A. Goddard, M. S. Gordon, W. J. Hehre, A. Klamt, H. F. Schaefer, M. W. Schmidt, C. D. Sherrill, D. G. Truhlar, A. Warshel, X. Xu, A. AspuruGuzik, R. Baer, A. T. Bell, N. A. Besley, J.-D. Chai, A. Dreuw, B. D. Dunietz, T. R. Furlani, S. R. Gwaltney, C.-P. Hsu, Y. Jung, J. Kong, D. S. Lambrecht, W. Liang, C. Ochsenfeld, V. A. Rassolov, L. V. Slipchenko, J. E. Subotnik, T. Van Voorhis, J. M. Herbert, A. I. Krylov, P. M. W. Gill and M. Head-Gordon, Mol. Phys. 113, 184 (2015).

I. Powis, A. B. Trofimov, I. L. Badsyuk, D. M. P. Holland, A. W. Potts, L. Karlsson, Chem. Phys. 415, 291 (2013).

[58] A. W. Potts, D. M. P. Holland, I. Powis, L. Karlsson, A. B. Trofimov, I. L. Badsyuk, Chem. Phys. 415, 84 (2013).

[59] M. W. Schmidt, K. K. Baldridge, J. A. Boatz, S. T. Elbert, M. S. Gordon, J. H. Jensen, S. Koseki, N. Matsunaga, K. A. Nguyen, S. J. Su, T. L. Windus, M. Dupuis, and J. A. Montgomery, J. Comput. Chem. 14, 1347 (1993).

[60] M. S. Gordon and M. W. Schmidt, in Theory and Applications of Computational Chemistry, edited by C. E. Dykstra, G. Frenking, K. S. Kim, and G. E. Scuseria (Elsevier, Amsterdam, 2005), p. 1167.

[61] G. Herzberg, Molecular Spectra and Molecular Structure II: Infrared and Raman Spectra of Polyatomic Molecules (Van Nostrand Reinhold, New York, 1945). 
[62] M. J. Frisch, G. W. Trucks, H. B. Schlegel, G. E. Scuseria, M. A. Robb, J. R. Cheeseman, G. Scalmani, V. Barone, G. A. Petersson, H. Nakatsuji, X. Li, M. Caricato, A. V. Marenich, J. Bloino, B. G. Janesko, R. Gomperts, B. Mennucci, H. P. Hratchian, J. V. Ortiz, A. F. Izmaylov, J. L. Sonnenberg, D. Williams-Young, F. Ding, F. Lipparini, F. Egidi, J. Goings, B. Peng, A. Petrone, T. Henderson, D. Ranasinghe, V. G. Zakrzewski, J. Gao, N. Rega, G. Zheng, W. Liang, M. Hada, M. Ehara, K. Toyota, R. Fukuda, J. Hasegawa, M. Ishida, T. Nakajima, Y. Honda, O. Kitao, H. Nakai, T. Vreven, K. Throssell, J. J. A. Montgomery, J. E. Peralta, F. Ogliaro, M. Bearpark, J. J. Heyd, E. Brothers, K. N. Kudin, V. N. Staroverov, T. A. Keith, R. Kobayashi, J. Normand, K. Raghavachari, A. Rendell, J. C. Burant, S. S. Iyengar, J. Tomasi, M. Cossi, J. M. Millam, M. Klene, C. Adamo, R. Cammi, J. W. Ochterski, R. L. Martin, K. Morokuma, O. Farkas, J. B. Foresman, and D. J. Fox, Gaussian 16 Revision A.03 (Gaussian Inc., Wallingford, CT, 2016).

[63] P. Downie and I Powis, J. Chem. Phys. 111, 4535 (1999).

[64] M. Staniforth, S. Daly, K. L. Reid, and I. Powis, J. Chem. Phys. 139, 064304 (2013).

[65] J. C. Slater, The Self-Consistent Field for Molecules and Solids (McGraw-Hill, New York, 1974).

[66] J. G. Norman, Jr., Mol. Phys. 31, 1191 (1976).

[67] G. Wendin, Breakdown of one-electron pictures in photoelectron spectra. In: Breakdown of the One-Electron Pictures in Photoelectron Spectra. Structure and Bonding, vol 45. (Springer, Berlin 1982).

[68] A. B. Trofimov, J. Schirmer, D. M. P. Holland, L. Karlsson, R. Maripuu, K. Siegbahn, and A. W. Potts, Chem. Phys. 263, 167 (2001).

[69] A. W. Potts, A. B. Trofimov, J. Schirmer, D. M. P. Holland, and L. Karlsson, Chem. Phys. 271, 337 (2001).

[70] A. B. Trofimov, J. Schirmer, D. M. P. Holland, A. W. Potts, L. Karlsson, R. Maripuu, and K. Siegbahn, J. Phys. B: At. Mol. Phys. 35, 5051 (2002).

[71] I. L. Zaytseva, A. B. Trofimov, J. Schirmer, O. Plekan, V. Feyer, R. Richter, M. Coreno, and K. C Prince, J. Phys. Chem. A 113, 15142 (2009). 
[72] H. Koch, O. Christiansen, P. Jørgensen, and J. Olsen, Chem. Phys. Lett. 244, 75 (1995).

[73] O. Christiansen, H. Koch, P. Jørgensen, and J. Olsen, Chem. Phys. Let, 256, 185 (1996).

[74] E. Larsen, K. Hald, J. Olsen, and P. Jørgensen, J. Chem. Phys. 115, 3015 (2001).

[75] J. P. Merrick, D. Moran, and L. Radom, J. Phys. Chem. A 111, 11683 (2007).

[76] I. Powis, R. C. Menzies, D. M. P. Holland, A. B. Trofimov, A. D. Skitnevskaya, E. V. Gromov, E. Antonsson, M. Patanen, C. Nicolas, and C. Miron, J. Chem. Phys. 149, 074305 (2018).

[77] A. B. Trofimov, I. Powis, R. C. Menzies, D. M. P. Holland, E. Antonsson, M. Patanen, C. Nicolas, C. Miron, A. D. Skitnevskaya, E. V. Gromov, and H. Köppel, J. Chem. Phys. 149, 074306 (2018).

[78] A. T. Duran, I. Powis, D. M. P. Holland, C. Nicolas, J. Bozek, A. B. Trofimov, E. K. Grigoricheva, and A. D. Skitnevskaya, J. Chem. Phys. 154, 094303 (2021).

[79] J. L. Dehmer, D. Dill, and S. Wallace, Phys. Rev. Lett. 43, 1005 (1979).

[80] D. M. P. Holland, L. Karlsson, and W. von Niessen, J. Electron Spectrosc. Relat. Phenom. 113, 221 (2001).

[81] D. M. P. Holland, I. Powis, G. Öhrwall, and W. von Niessen, Chem. Phys. 326, 535 (2006).

[82] I. Powis, I. L. Zaytseva, A. B. Trofimov, J. Schirmer, D. M. P. Holland, A. W. Potts, and L. Karlsson, J. Phys. B: At. Mol. Phys. 40, 2019 (2007)

[83] W. Domcke, Phys. Scripta, 19, 11 (1979). 
TABLE I. Energy ranges used to analyse the experimental photoelectron spectra.

\begin{tabular}{ccc}
\hline \hline Region & Energy range $(\mathrm{eV})$ & Orbitals $^{\mathrm{a}}$ \\
\hline 1 & $8.60-9.70$ & $3 \mathrm{a}^{\prime \prime}$ \\
2 & $9.75-10.50$ & $15 \mathrm{a}^{\prime}, 2 \mathrm{a}^{\prime \prime}$ \\
3 & $10.50-11.30$ & $15 \mathrm{a}^{\prime}, 2 \mathrm{a}^{\prime \prime}$ \\
4 & $13.10-13.70$ & $1 \mathrm{a}^{\prime \prime}, 14 \mathrm{a}^{\prime}$ \\
5 & $13.70-14.30$ & $14 \mathrm{a}^{\prime}, 1 \mathrm{a}^{\prime \prime}$ \\
6 & $14.30-15.10$ & $1 \mathrm{a}^{\prime \prime}, 13 \mathrm{a}^{\prime}, 12 \mathrm{a}^{\prime}$ \\
7 & $15.10-16.30$ & $13 \mathrm{a}^{\prime}, 12 \mathrm{a}^{\prime}$ \\
8 & $17.30-18.30$ & $11 \mathrm{a}^{\prime}, 10 \mathrm{a}^{\prime}, 9 \mathrm{a}^{\prime}$ \\
9 & $18.30-18.90$ & $11 \mathrm{a}^{\prime}, 10 \mathrm{a}^{\prime}, 9 \mathrm{a}^{\prime}$ \\
10 & $18.90-19.60$ & $11 \mathrm{a}^{\prime}, 10 \mathrm{a}^{\prime}, 9 \mathrm{a}^{\prime}$ \\
11 & $19.60-21.00$ & $11 \mathrm{a}^{\prime}, 10 \mathrm{a}^{\prime}, 9 \mathrm{a}^{\prime}$ \\
& & \\
\hline \hline
\end{tabular}

${ }^{\text {a }}$ Orbitals possibly contributing to the observed electron intensity in a specific energy range. 
TABLE II. Vertical ionization energies (eV) of the outer valence transitions in imidazole. These are computed using the HF/Koopmans', OVGF, IP-ADC(3), Dyson ADC(3), EOM-IP-CCSD and CC3 methods, as well as their best theoretical estimates (BTE) in comparison with the experimental data. For the $\operatorname{ADC}(3)$ schemes, relative intensities (pole strengths) are also presented in parentheses. ${ }^{a}$

\begin{tabular}{|c|c|c|c|c|c|c|c|c|c|c|c|c|}
\hline State & \multicolumn{2}{|c|}{ MO } & \multirow{2}{*}{$\begin{array}{c}\mathrm{HF} \\
8.63\end{array}$} & \multirow{2}{*}{$\frac{\text { OVGF }}{8.82}$} & \multicolumn{2}{|c|}{ IP-ADC(3) } & \multicolumn{2}{|c|}{ Dyson ADC(3) } & \multirow{2}{*}{$\begin{array}{c}\text { CCSD } \\
8.91\end{array}$} & \multirow{2}{*}{$\begin{array}{l}\mathrm{CC} 3 \\
8.85\end{array}$} & \multirow{2}{*}{$\frac{\text { BTE }^{b}}{9.02}$} & \multirow{2}{*}{$\frac{\text { Expt. }^{c}}{9.1}$} \\
\hline$X^{2} \mathrm{~A}^{\prime \prime}$ & $3 a^{\prime \prime}$ & $\pi$ & & & 8.85 & $(0.90)$ & 8.89 & $(0.89)$ & & & & \\
\hline$A^{2} \mathrm{~A}^{\prime}$ & $15 a^{\prime}$ & $\mathrm{n} \sigma$ & 11.85 & 10.27 & 10.47 & $(0.89)$ & 10.56 & $(0.89)$ & 10.15 & 10.08 & 10.30 & \multirow{2}{*}{10.4} \\
\hline$B^{2} \mathrm{~A}^{\prime \prime}$ & $2 a^{\prime \prime}$ & $\pi$ & 10.85 & 10.25 & 10.34 & $(0.87)$ & 10.40 & $(0.87)$ & 10.32 & 10.30 & 10.51 & \\
\hline${ }^{2} \mathrm{~A}^{\prime \prime}$ & $1 a^{\prime \prime}$ & $\pi$ & - & - & 13.78 & $(0.21)$ & 13.90 & $(0.24)$ & - & 13.94 & - & \multirow{2}{*}{13.8} \\
\hline$C^{2} \mathrm{~A}^{\prime}$ & $14 a^{\prime}$ & $\sigma$ & 15.45 & 14.16 & 14.32 & $(0.89)$ & 14.34 & $(0.89)$ & 14.18 & 14.06 & 14.25 & \\
\hline$D^{2} \mathrm{~A}^{\prime \prime}$ & $1 a^{\prime \prime}$ & $\pi$ & 16.25 & 14.55 & 14.90 & $(0.50)$ & 15.03 & $(0.46)$ & 14.60 & - & 14.81 & \multirow{4}{*}{$\begin{array}{c}14.8- \\
15.3\end{array}$} \\
\hline$E^{2} \mathrm{~A}^{\prime}$ & $13 \mathrm{a}^{\prime}$ & $\sigma$ & 16.37 & 15.05 & 15.20 & $(0.76)$ & 15.23 & $(0.71)$ & 15.03 & 14.89 & 15.09 & \\
\hline${ }^{2} \mathrm{~A}^{\prime}$ & $13 \mathrm{a}^{\prime}$ & $\sigma$ & - & - & 15.26 & $(0.14)$ & 15.28 & $(0.20)$ & - & - & - & \\
\hline$F^{2} \mathrm{~A}^{\prime}$ & $12 \mathrm{a}^{\prime}$ & $\sigma$ & 16.51 & 15.10 & 15.32 & $(0.87)$ & 15.36 & $(0.88)$ & 15.14 & - & 15.33 & \\
\hline$G^{2} \mathrm{~A}^{\prime}$ & $11 a^{\prime}$ & $\sigma$ & 20.61 & 18.65 & 18.58 & $(0.57)$ & 18.62 & $(0.59)$ & 18.67 & 17.95 & 18.12 & 17.9 \\
\hline
\end{tabular}

${ }^{a}$ The cc-pVTZ basis set was employed everywhere except for the Dyson ADC(3) calculations which were performed using the cc-pVTZ basis on the second row atoms and the cc-pVDZ basis on the hydrogens.

${ }^{\mathrm{b}}$ The best theoretical estimates were obtained by adding the CBS correction for the cc-pVTZ basis (Table IV) to the CC3 values. In the case of the $D^{2} \mathrm{~A}^{\prime \prime}$ and $F^{2} \mathrm{~A}^{\prime}$ states where CC3 values could not be obtained, the correction is added to the EOM-IP-CCSD values.

${ }^{\mathrm{c}}$ As derived from the band maxima in the photoelectron spectrum recorded with a photon energy of $80 \mathrm{eV}$. For the $X^{2} \mathrm{Al}$ state, an approximate position of the centre of gravity for the band is given (which roughly corresponds to the vertical transition energy), the energy of the $0-0$ transition is $8.842 \mathrm{eV}$. 
TABLE III. Transitions originating from ionization of the $1 \mathrm{a}^{\prime \prime}$ orbital of imidazole: vertical ionization energy $(I E, \mathrm{eV})$, pole strength $(P$, a.u.), weight $(C$, percent) of the most important $1 h$ and $2 h-1 p$ configurations (specified) in the respective wavefunction, as calculated at the IP-ADC(3) level of theory, and their type. ${ }^{\mathrm{a}}$

\begin{tabular}{|c|c|c|c|c|}
\hline$I E$ & $P$ & $C(1 h)$ & $C(2 h-1 p)$ & Type \\
\hline 13.78 & 0.21 & $20\left(1 a^{\prime \prime}\right)^{-1}$ & $29\left(3 \mathrm{a}^{\prime \prime}\right)^{-2}\left(4 \mathrm{a}^{\prime \prime}\right)^{1}$ & shake-down \\
\hline 14.90 & 0.50 & $51\left(1 a^{\prime \prime}\right)^{-1}$ & $7\left(3 a^{\prime \prime}\right)^{-2}\left(4 a^{\prime \prime}\right)^{1}$ & main line \\
\hline 15.36 & 0.02 & $1\left(1 a^{\prime \prime}\right)^{-1}$ & $29\left(3 \mathrm{a}^{\prime \prime}\right)^{-2}\left(5 \mathrm{a}^{\prime \prime}\right)^{1}$ & shake-up \\
\hline 16.06 & 0.07 & $7\left(1 a^{\prime}\right)^{-1}$ & $17\left(2 a^{\prime \prime}\right)^{-1}(3 a ")^{-1}\left(4 a^{\prime \prime}\right)^{1}$ & shake-up \\
\hline 17.70 & 0.06 & $5\left(1 a^{\prime \prime}\right)^{-1}$ & $13\left(2 a^{\prime \prime}\right)^{-1}\left(3 a^{\prime \prime}\right)^{-1}\left(5 a^{\prime \prime}\right)^{1}$ & shake-up \\
\hline 19.56 & 0.06 & $6\left(1 a^{\prime \prime}\right)^{-1}$ & $13\left(2 a^{\prime \prime}\right)^{-2}\left(4 a^{\prime \prime}\right)^{1}$ & shake-up \\
\hline
\end{tabular}

${ }^{a}$ Only transitions with $\mathrm{P} \geq 0.01$ are shown. 
TABLE IV. Vertical ionization energies (eV) of imidazole computed using the EOM-IP-CCSD method and series of the cc-pVnZ and aug-cc-pVmZ basis sets ( $n=\mathrm{D}, \mathrm{T}, \mathrm{Q}, 5 ; m=\mathrm{D}, \mathrm{T}, \mathrm{Q})$, as well as the estimates for the complete basis set limit, $\Delta(\infty)$.

\begin{tabular}{cccccccccc}
\hline \hline State & MO & cc-pVDZ & $\begin{array}{c}\text { aug-cc- } \\
\text { pVDZ }\end{array}$ & cc-pVTZ & $\begin{array}{c}\text { aug-cc- } \\
\text { pVTZ }\end{array}$ & cc-pVQZ & $\begin{array}{c}\text { aug-cc- } \\
\text { pVQZ }\end{array}$ & cc-pV5Z $^{\Delta(\infty){ }^{\text {a }}}$ \\
\hline$X^{2} \mathrm{~A}^{\prime \prime}$ & $3 \mathrm{a}^{\prime \prime}$ & 8.64 & 8.85 & 8.91 & 9.00 & 9.01 & 9.05 & 9.06 & 9.08 \\
$A^{2} \mathrm{~A}^{\prime}$ & $15 \mathrm{a}^{\prime}$ & 9.79 & 10.07 & 10.15 & 10.26 & 10.29 & 10.33 & 10.34 & 10.37 \\
$B^{2} \mathrm{~A}^{\prime \prime}$ & $2 \mathrm{a}^{\prime \prime}$ & 10.00 & 10.24 & 10.32 & 10.43 & 10.45 & 10.49 & 10.50 & 10.53 \\
$C^{2} \mathrm{~A}^{\prime}$ & $14 \mathrm{a}^{\prime}$ & 13.95 & 14.14 & 14.18 & 14.27 & 14.29 & 14.33 & 14.34 & 14.37 \\
$D^{2} \mathrm{~A}^{\prime \prime}$ & $1 \mathrm{a}^{\prime \prime}$ & 14.33 & 14.53 & 14.60 & 14.70 & 14.72 & 14.77 & 14.77 & 14.81 \\
$E^{2} \mathrm{~A}^{\prime}$ & $13 \mathrm{a}^{\prime}$ & 14.80 & 14.98 & 15.03 & 15.11 & 15.14 & 15.17 & 15.18 & 15.23 \\
$F^{2} \mathrm{~A}^{\prime}$ & $12 \mathrm{a}^{\prime}$ & 14.92 & 15.09 & 15.14 & 15.22 & 15.24 & 15.28 & 15.29 & 15.33 \\
$G^{2} \mathrm{~A}^{\prime}$ & $11 \mathrm{a}^{\prime}$ & 18.46 & 18.62 & 18.67 & 18.74 & 18.77 & 18.80 & 18.81 & 18.84 \\
\hline \hline
\end{tabular}

${ }^{a}$ Mean of the cc-pVnZ and aug-cc-pVnZ basis set limits $(n \rightarrow \infty)$, see text for details. 


\section{FIGURE CAPTIONS}

Figure 1

The outer valence molecular orbitals of imidazole (iso-surface HF density plots). The top left panel indicates the molecular orientation used for these plots, and the atomic numbering. The nitrogen, carbon and hydrogen atoms are coded blue, grey and white, respectively.

Figure 2

The complete inner and outer valence region photoelectron spectrum of imidazole: (a) the experimental spectrum recorded with parallel linear polarization using a photon energy of 80 $\mathrm{eV}$ (the sharp feature seen at $12.62 \mathrm{eV}$ binding energy is a residual trace of $\mathrm{H}_{2} \mathrm{O}$ in the sample); (b) simulated spectral profile using $\operatorname{ADC}(3)$ calculated transition energies and pole strengths (Table II), convolved with a $0.55 \mathrm{eV}$ FWHM Gaussian shaping function.

Figure 3

An outer valence photoelectron spectrum of imidazole, recorded with a photon energy of 24 $\mathrm{eV}$. Included in the figure are normalized measurements made with the linear polarization axis set either parallel or perpendicular to the spectrometer detection axis, showing the different intensity distributions. The photoelectron anisotropy parameter $\beta$ derived from these spectra, evaluated point by point across the spectrum is also plotted. (The plotting of $\beta$ is suppressed in regions of low photoelectron intensity since here the form of Eq. 2 greatly amplifies the statistical noise in the weak background baseline). The green horizontal bars mark the regions (R1 - R11) used to analyse the spectra. The binding energy ranges corresponding to these regions are listed in Table I. At the bottom are marked the best theoretical estimates (BTE) obtained by applying the complete basis set extrapolation estimate to the CC3/cc-pVTZ calculations (Table IV).

Figure 4

The magic angle $X^{2} \mathrm{~A}^{\prime \prime}$ state photoelectron spectrum of imidazole recorded using a photon energy of $24 \mathrm{eV}$. Also shown is a 300K FC simulated spectrum with the calculated B3LYP/cc- 
pVTZ harmonic frequencies scaled by a factor of $0.97 .{ }^{75}$ Its vibrationless origin is set at a binding energy of $8.842 \mathrm{eV}$. The intensity of both the experimental and the simulated spectra are arbitrarily scaled for plotting. Assignments of the most intense peaks in the stick spectrum are marked in the figure. Also shown is the $\beta$ anisotropy parameter curve obtained across this band.

\section{Figure 5}

The $2^{\text {nd }}$ band of the magic angle photoelectron spectrum of imidazole, recorded with a photon energy of $24 \mathrm{eV}$ : (a) the $\beta$-parameter derived from the parallel and perpendicular polarization recordings (Eq. 2) and the BTE values for the vertical 15a' and 2a" ionizations; (b) comparison with TD-B3LYP/cc-pVDZ FC simulation for 15a' ionization; (c) similar comparison for $2 \mathrm{a}^{\prime \prime}$ ionization with the origin set $0.21 \mathrm{eV}$ higher as suggested by the difference in the $15 \mathrm{a}^{\prime}$ and $2 \mathrm{a}^{\prime \prime}$ BTE binding energies. The harmonic frequencies in the simulations shown in (b) and (c) have been scaled by factors of 1.17 and 0.90 respectively. The principal vibrational excitations are marked on the figure.

\section{Figure 6}

The $3^{\text {rd }}$ band of the magic angle photoelectron spectrum of imidazole, recorded with a photon energy of $24 \mathrm{eV}$, and the $\beta$-parameter measurement across this region. The energies of the BTE vertical ionization estimates are marked at the bottom, along with the $\left(1 a^{\prime \prime}\right)$ and $\left(13 a^{\prime}\right)$ satellite transitions. A TD-B3LYP/cc-pVDZ FC simulation for the lowest $14 \mathrm{a}^{\prime}$ ionization, with harmonic frequencies scaled by a factor 1.2 is included for comparison.

\section{Figure 7}

The $4^{\text {th }}$ band of the magic angle photoelectron spectrum of imidazole, recorded with a photon energy of $24 \mathrm{eV}$, and the $\beta$-parameter measurement across this region. No vibrational simulations were attempted for this region (see text). 
Figure 8

The experimental and theoretical intensity branching ratios for the first, second and third photoelectron bands, corresponding to the $3 a^{\prime \prime},\left(15 a^{\prime}+2 a^{\prime \prime}\right)$, and (1 $\left.a^{\prime \prime}, 14 a^{\prime}, 13 a^{\prime}, 12 a^{\prime}\right)$ orbitals. The theoretical curves for each band were obtained by summing the CMS-X $\alpha$ photoionization partial cross sections corresponding to the orbitals assigned to that photoelectron band.

Figure 9

A comparison between the experimental and theoretically predicted photoelectron anisotropy parameters, $\beta$, for: (a) the first photoelectron band due to the $\left(3 \mathrm{a}^{\prime \prime}\right)^{-1} X^{2} \mathrm{~A}^{\prime \prime}$ state; (b) the second photoelectron band due to the $\left(15 \mathrm{a}^{\prime}\right)^{-1} A^{2} \mathrm{~A}^{\prime}$ and $\left(2 \mathrm{a}^{\prime \prime}\right)^{-1} B^{2} \mathrm{~A}^{\prime \prime}$ states. The binding energy regions used to sample the experimental data are defined in Table I.

Figure 10

A comparison between the experimental and theoretically predicted photoelectron anisotropy parameters, $\beta$, for ionizations contributing to the third photoelectron band (regions $4-7$, see Table I and Fig. 3). The $\left(14 \mathrm{a}^{\prime}\right)^{-1} C^{2} \mathrm{~A}^{\prime},\left(1 \mathrm{a}^{\prime \prime}\right)^{-1} D^{2} \mathrm{~A}^{\prime \prime},\left(13 \mathrm{a}^{\prime}\right)^{-1} E^{2} \mathrm{~A}^{\prime}$ and $\left(12 \mathrm{a}^{\prime}\right)^{-1} F^{2} \mathrm{~A}^{\prime}$ states lie in these regions (Table II), see text for details.

Figure 11

A comparison between the experimental and theoretically predicted photoelectron anisotropy parameters, $\beta$, for ionizations contributing to the fourth photoelectron band (regions $8-11$, see Table I and Fig. 3). CMS-Xa results are shown for the $11 a^{\prime}$ and $10 a^{\prime}$ orbitals. 


\section{FIGURES}

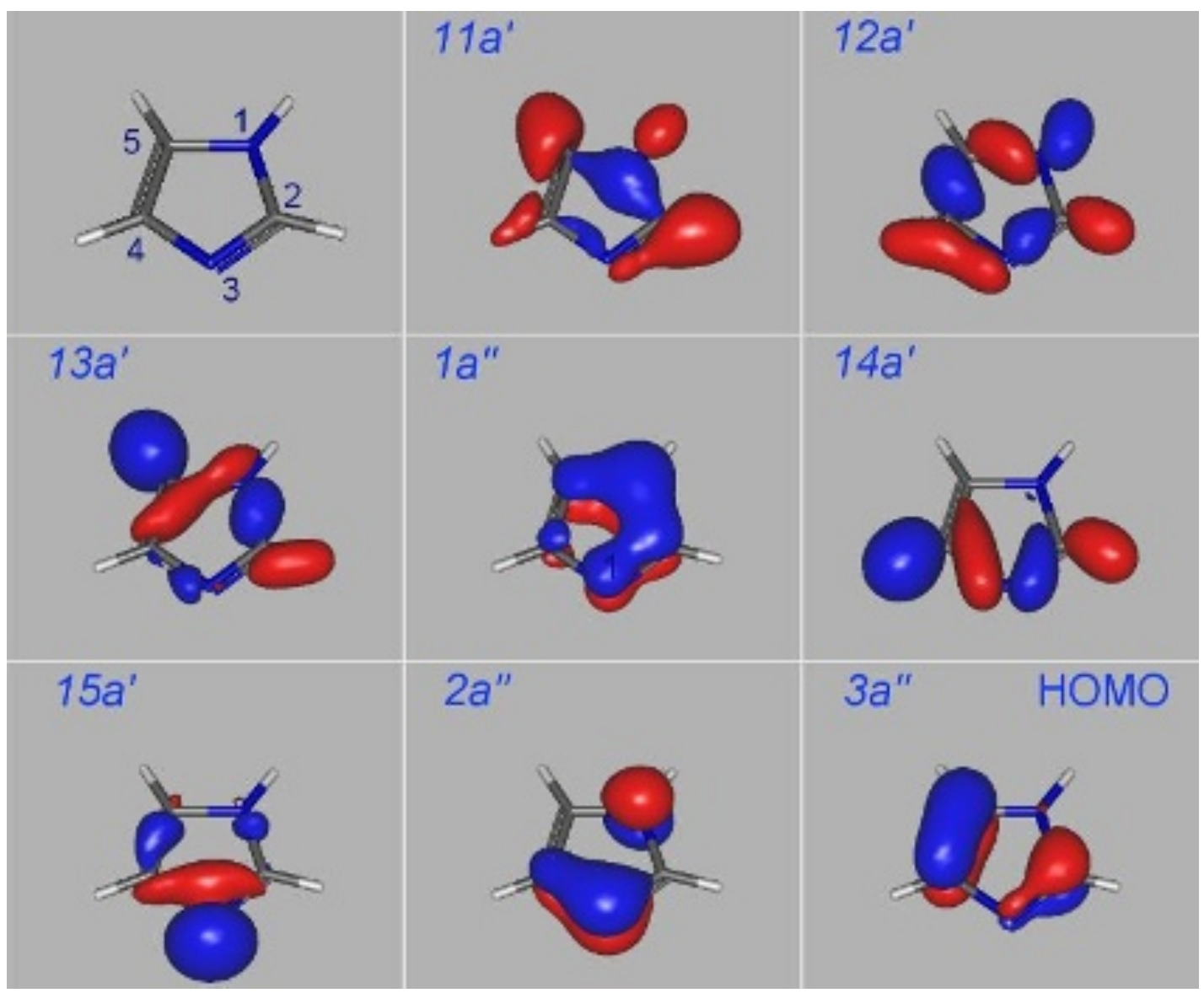

Figure 1 


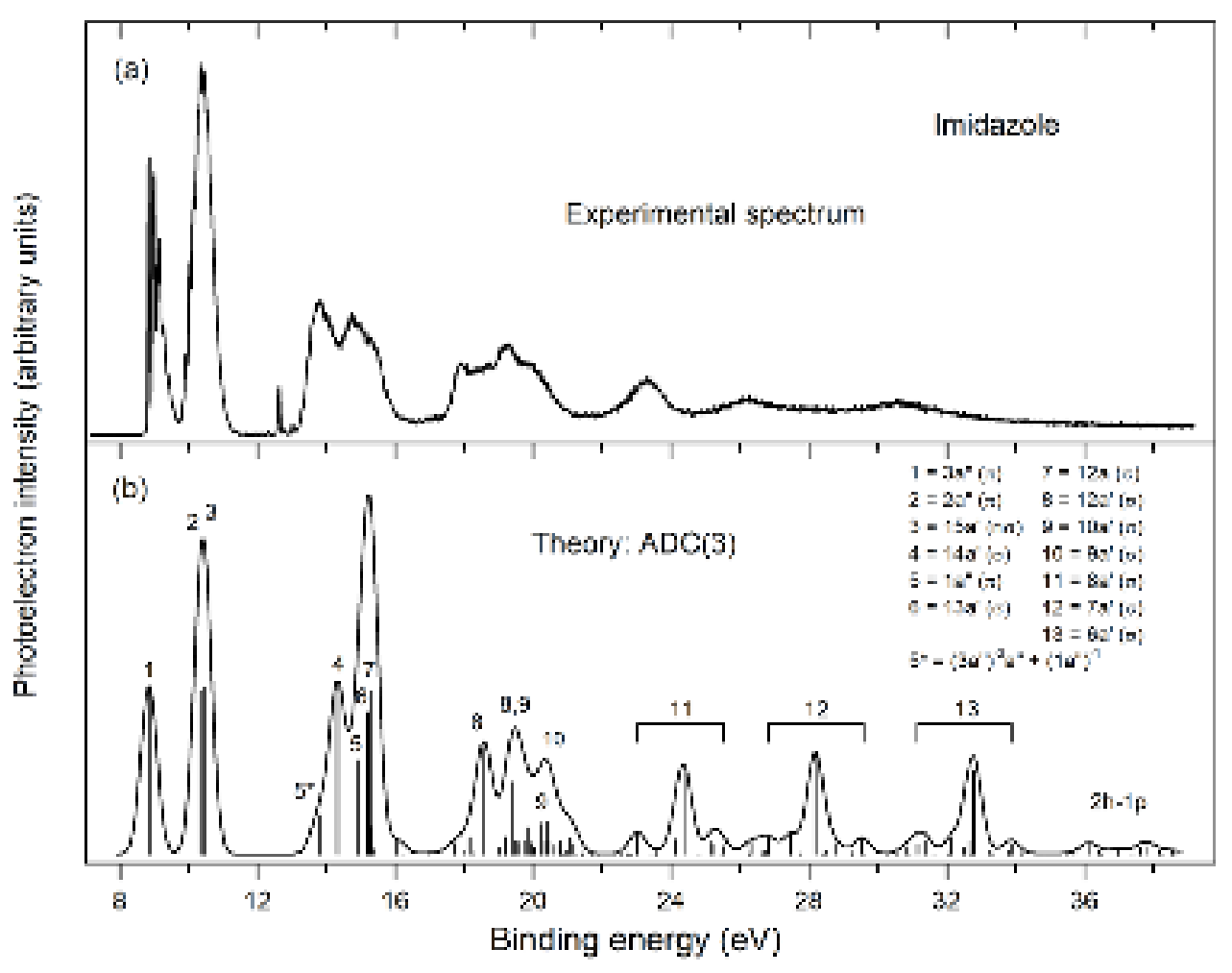

Figure 2 




Figure 3 


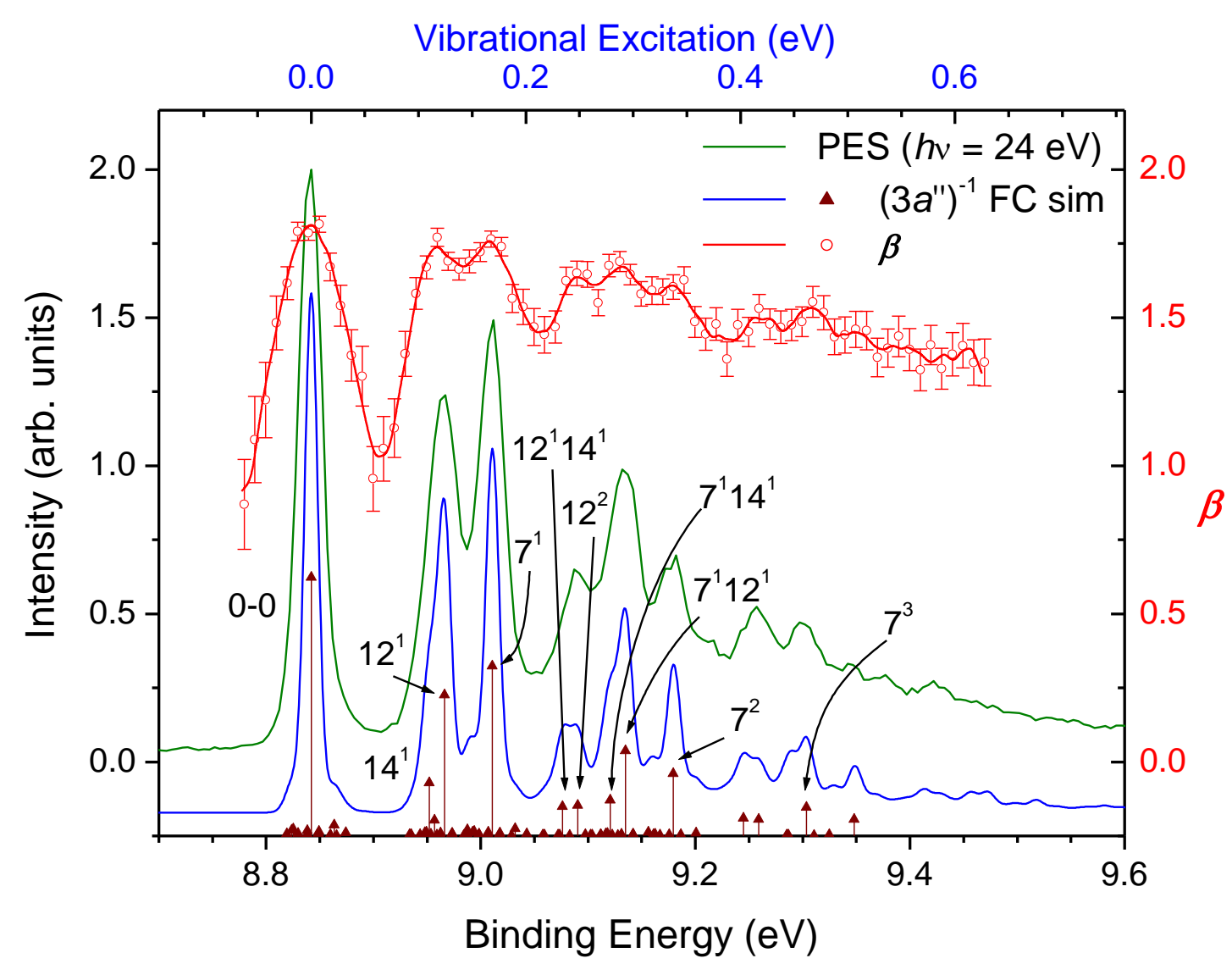

Figure 4 


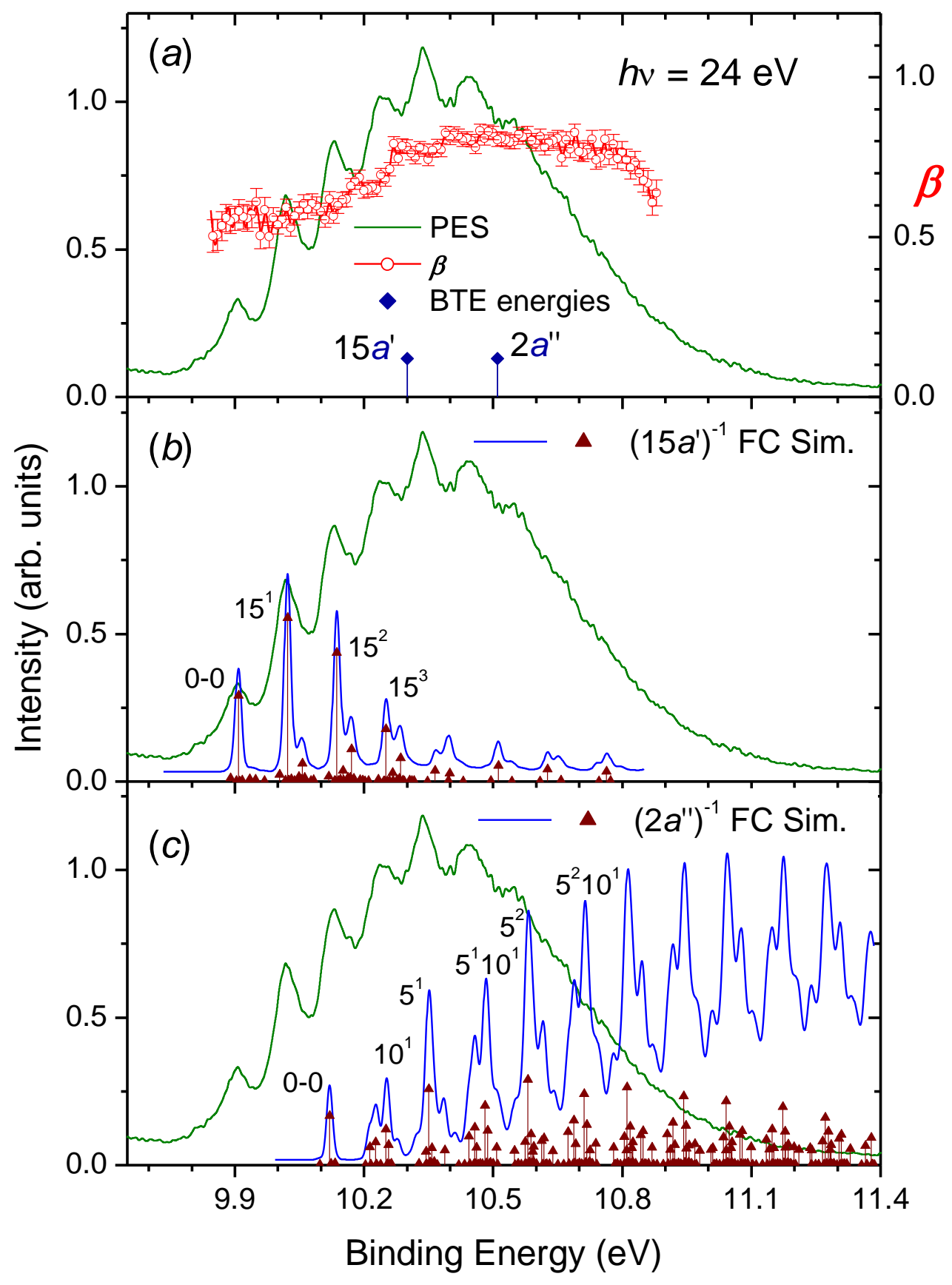

Figure 5 


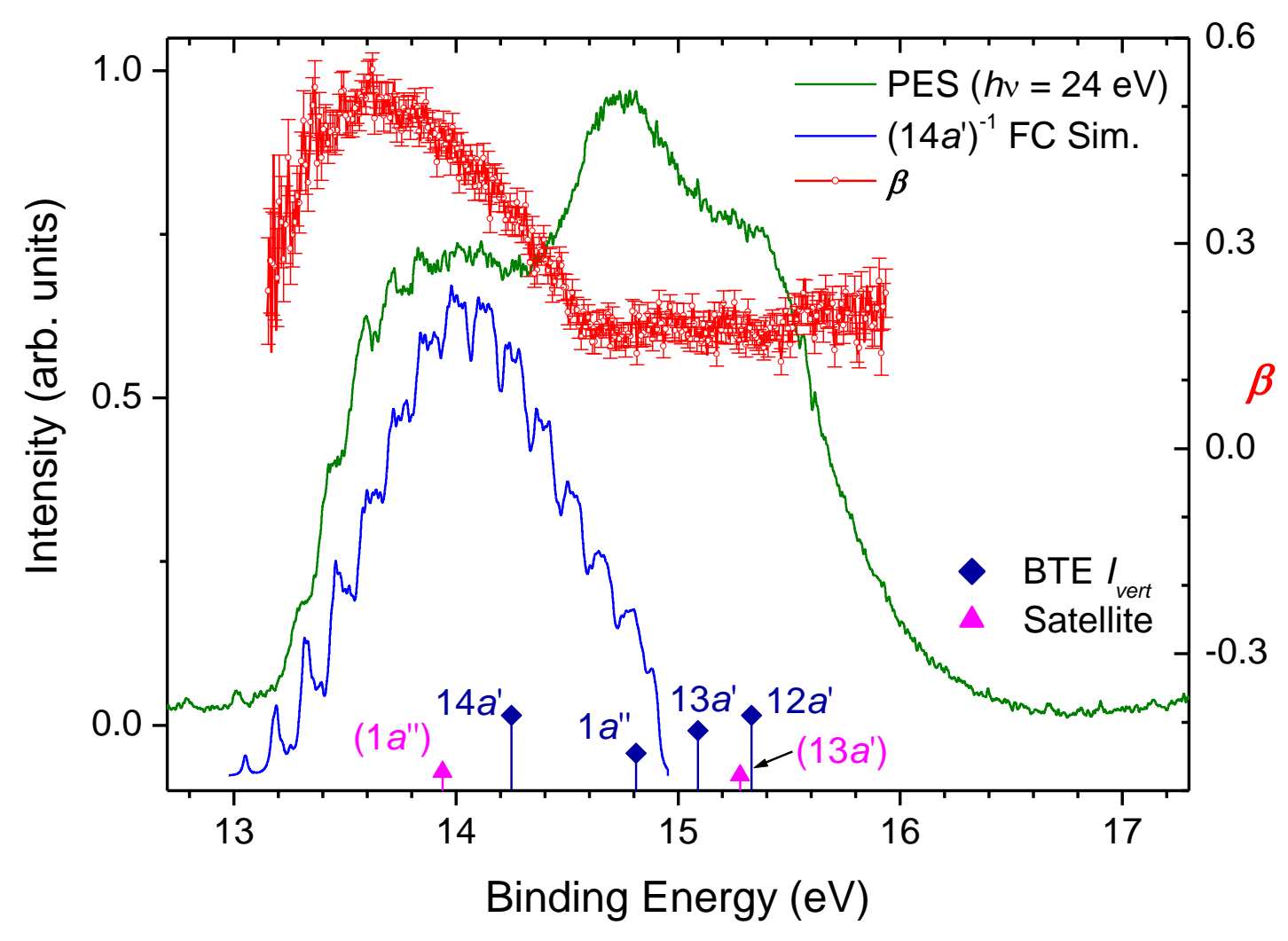

Figure 6 


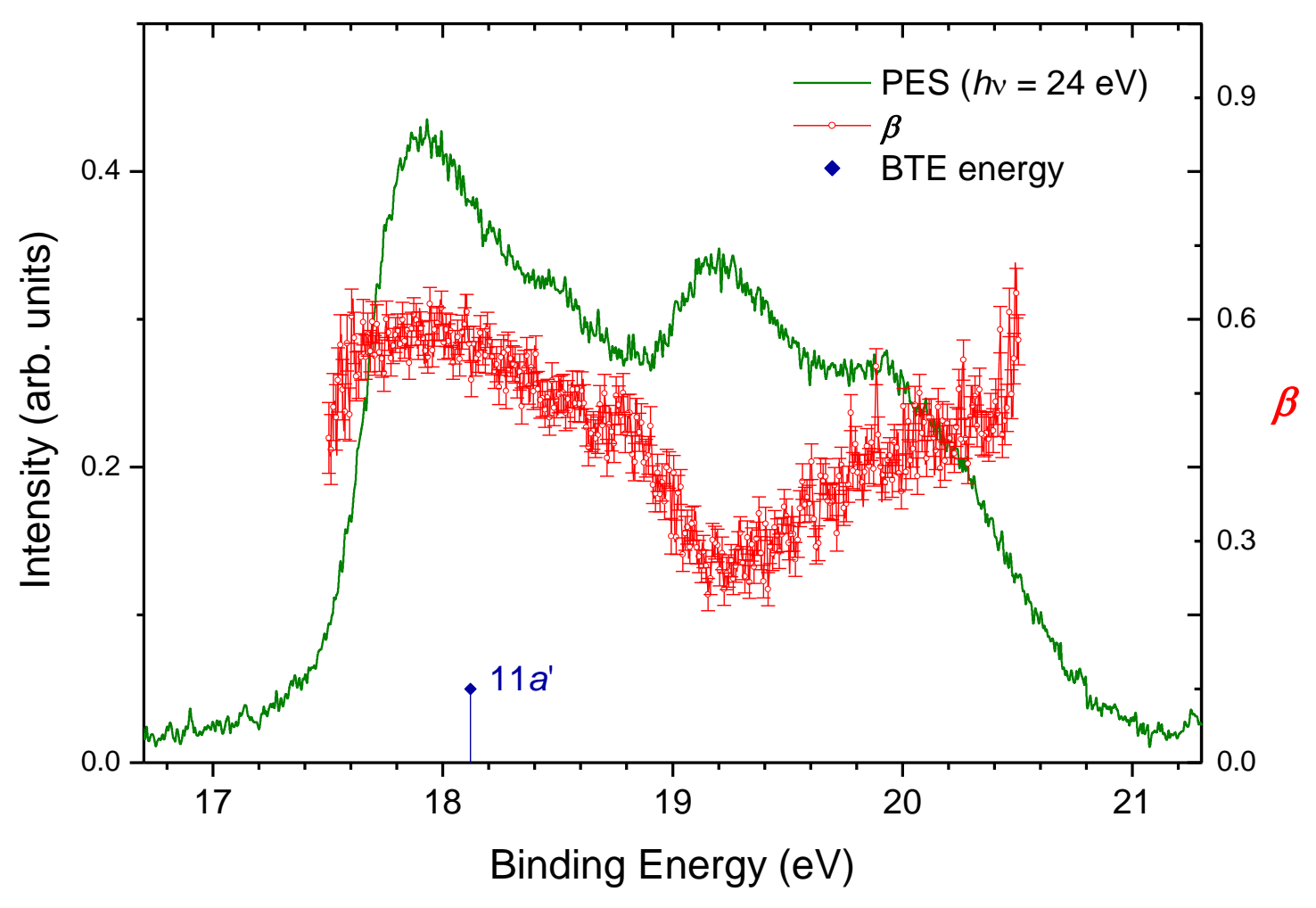

Figure 7 




Figure 8 


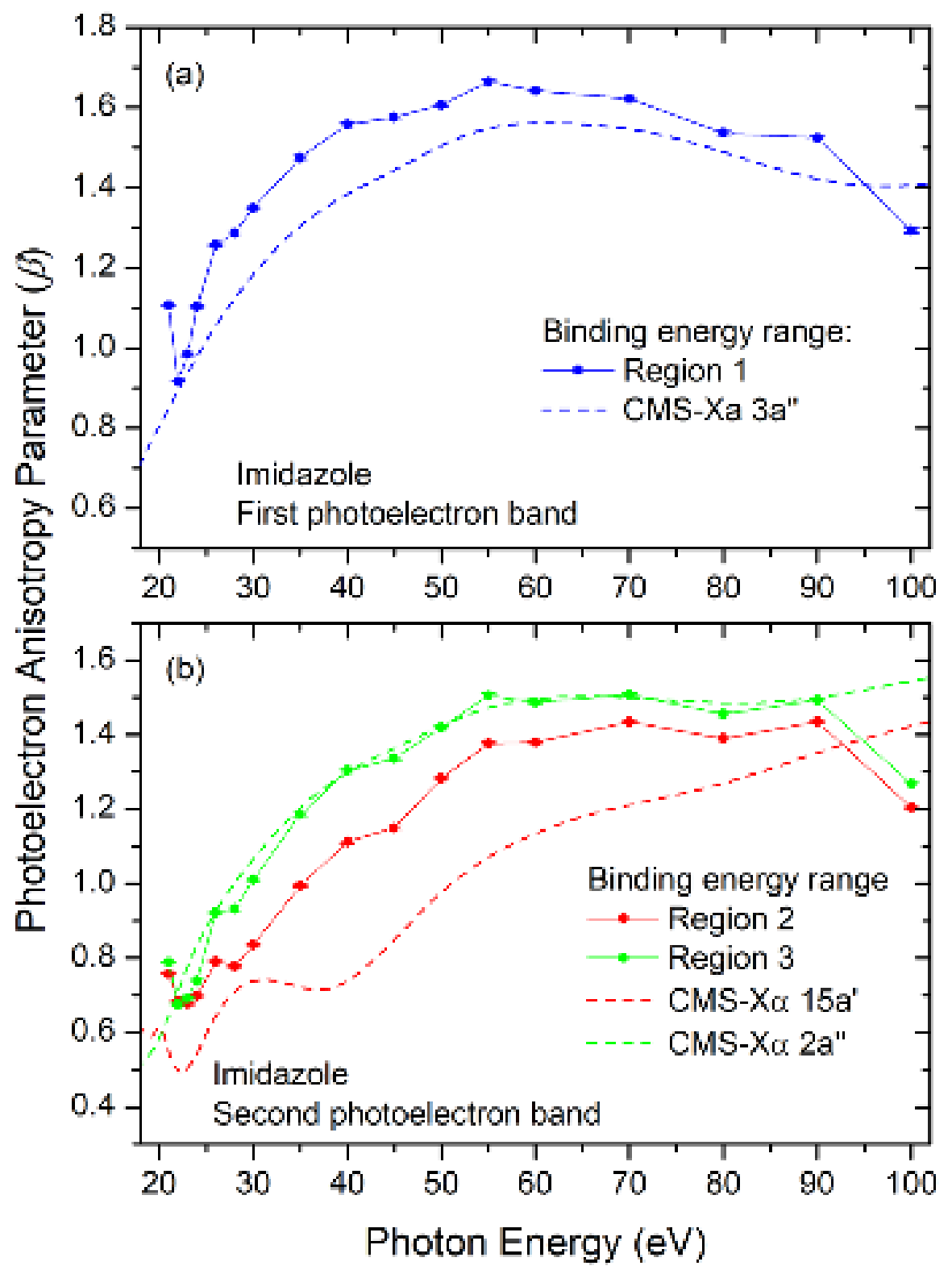

Figure 9 


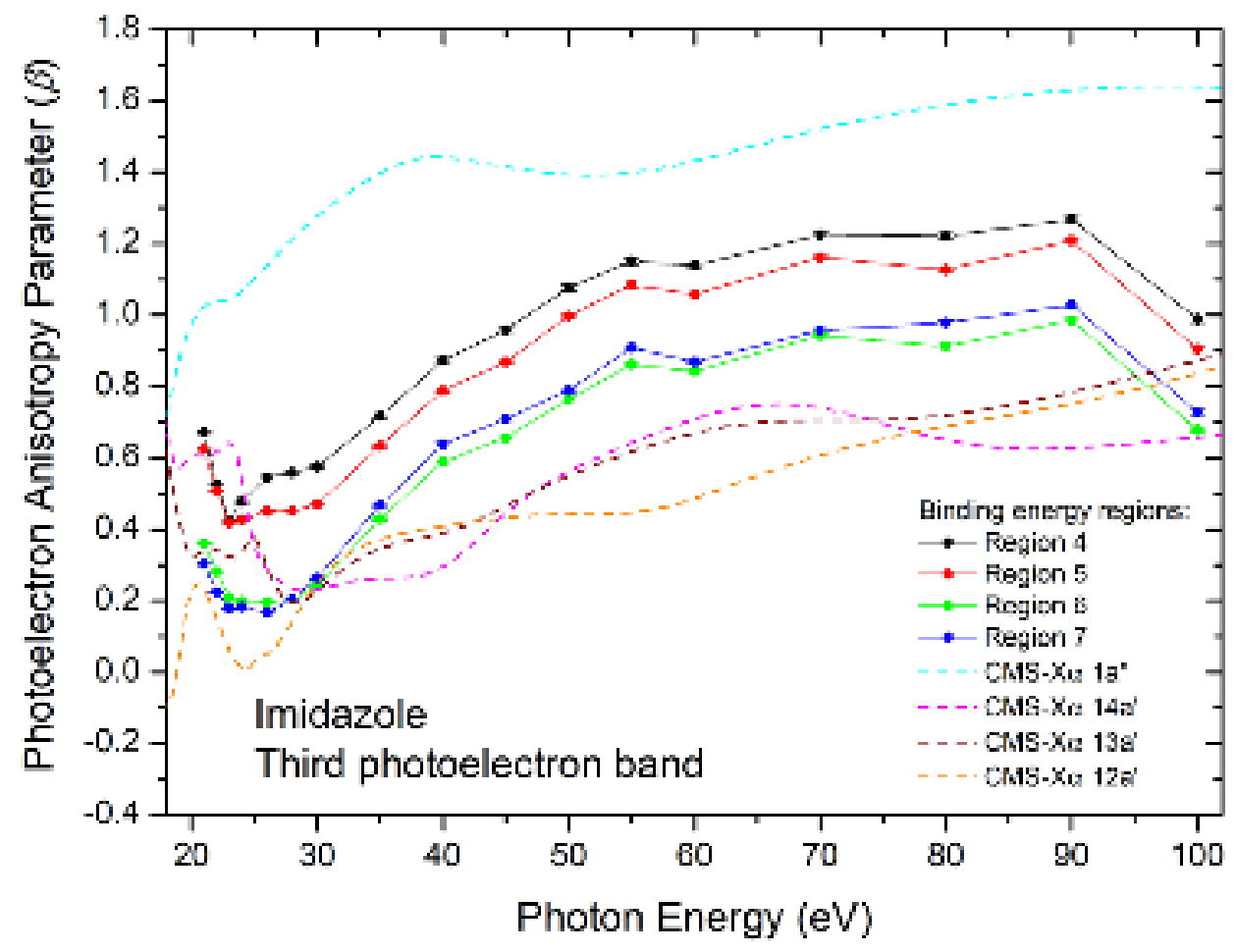

Figure 10 




Figure 11 
$11 a^{\circ}$

$12 a^{\circ}$

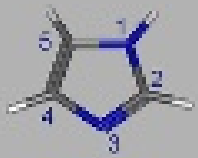

$138^{\circ}$

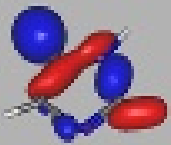

15a'

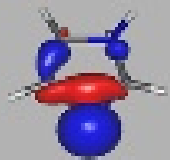

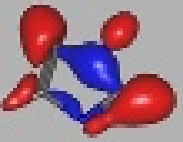

$1 a^{n}$

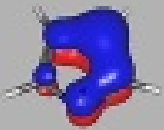

$2 a^{\prime \prime}$

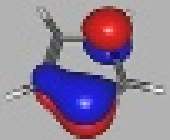

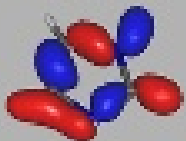

$14 a^{\circ}$

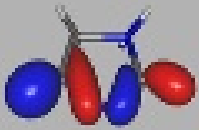

$3 a^{\prime \prime}$ HOMO

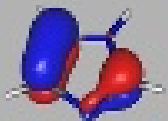




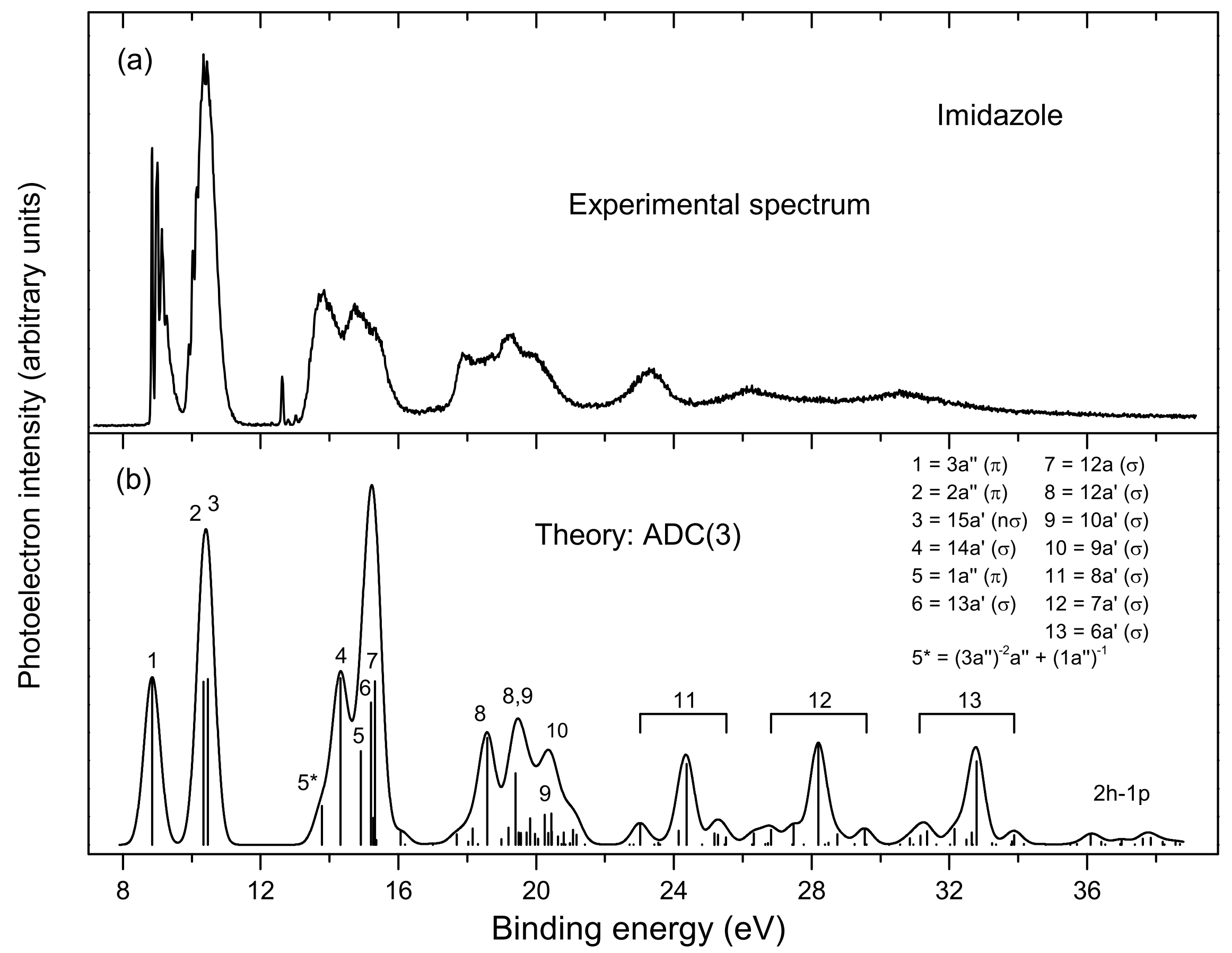




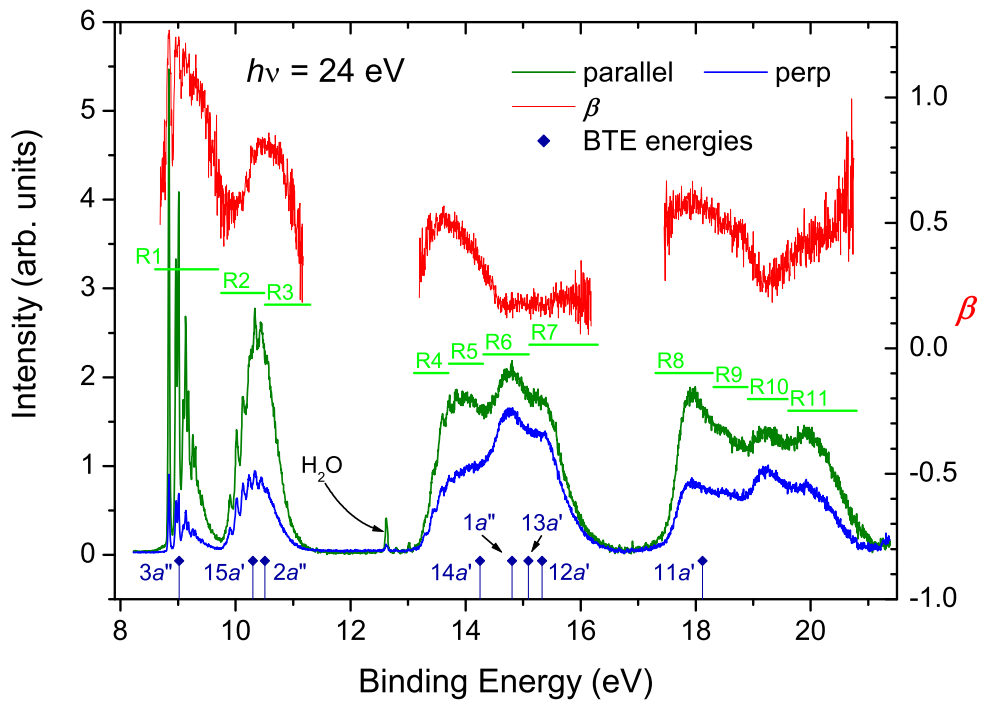




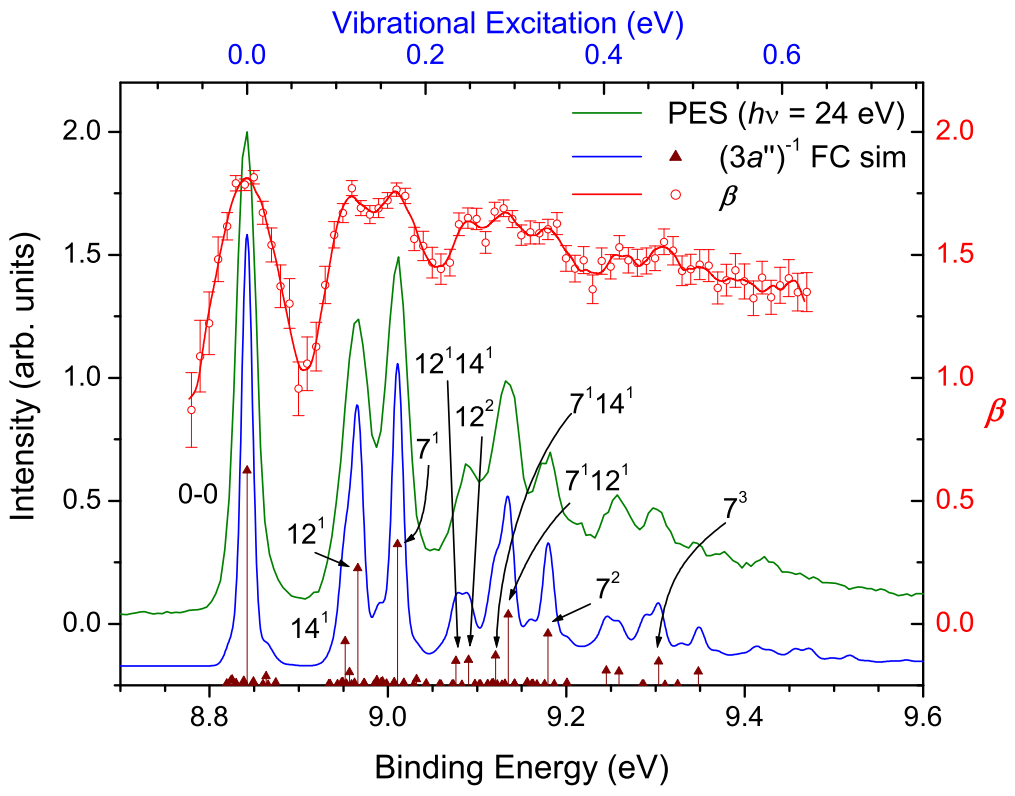




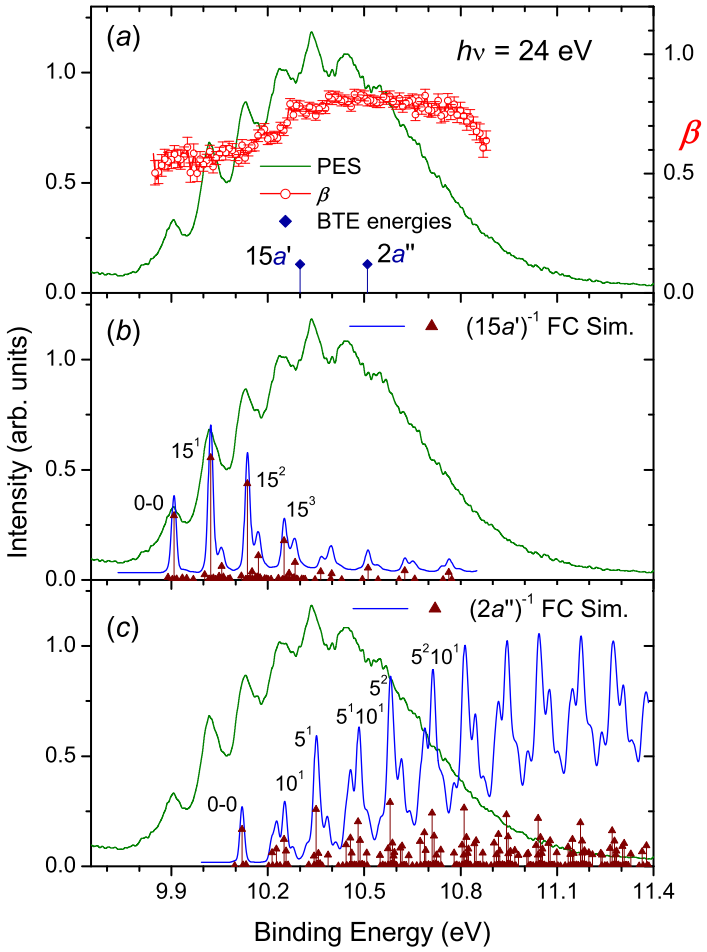




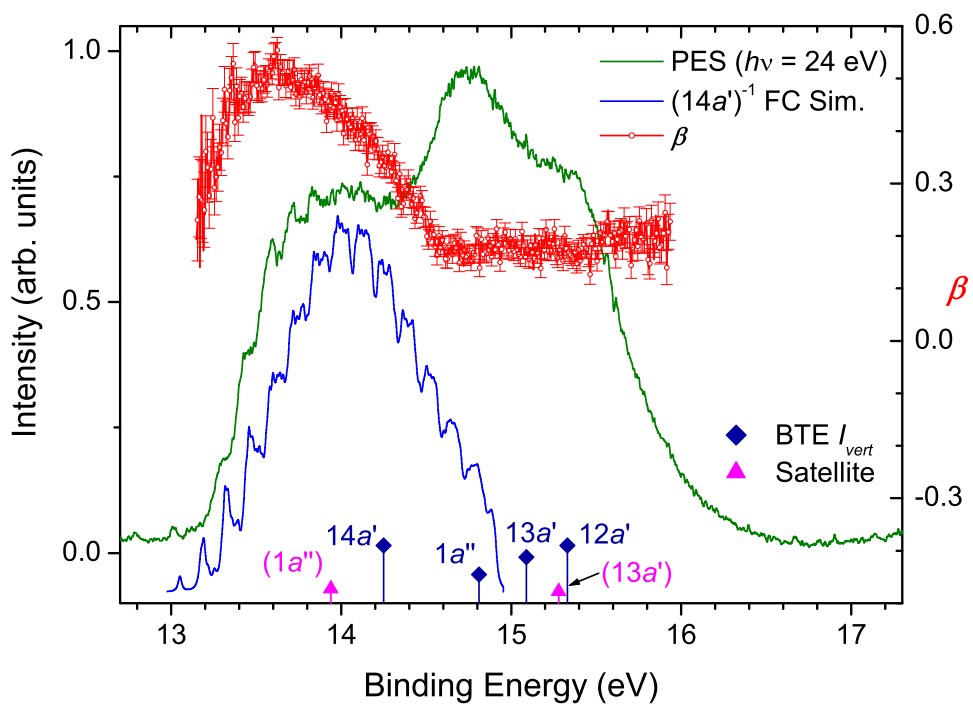









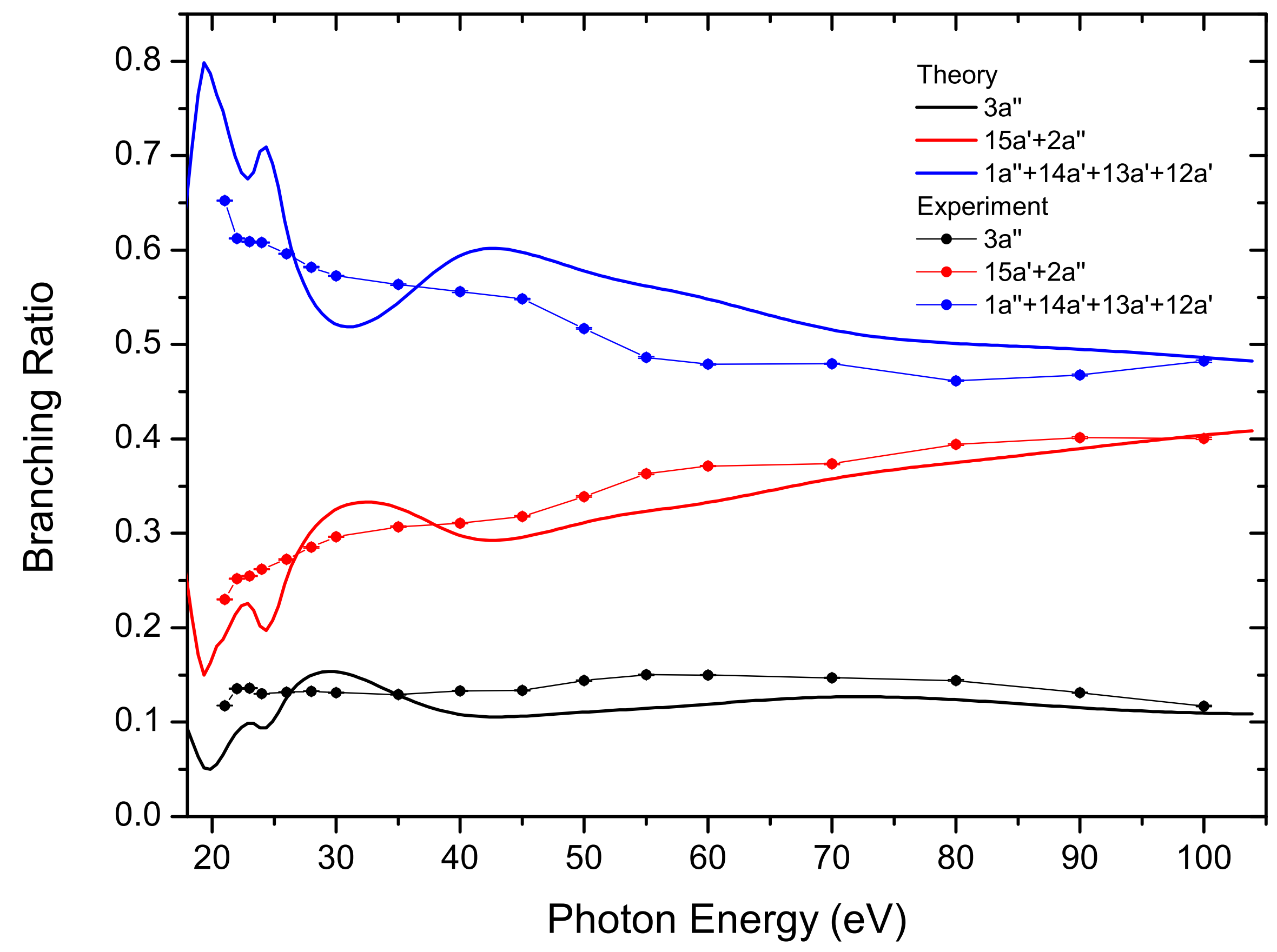




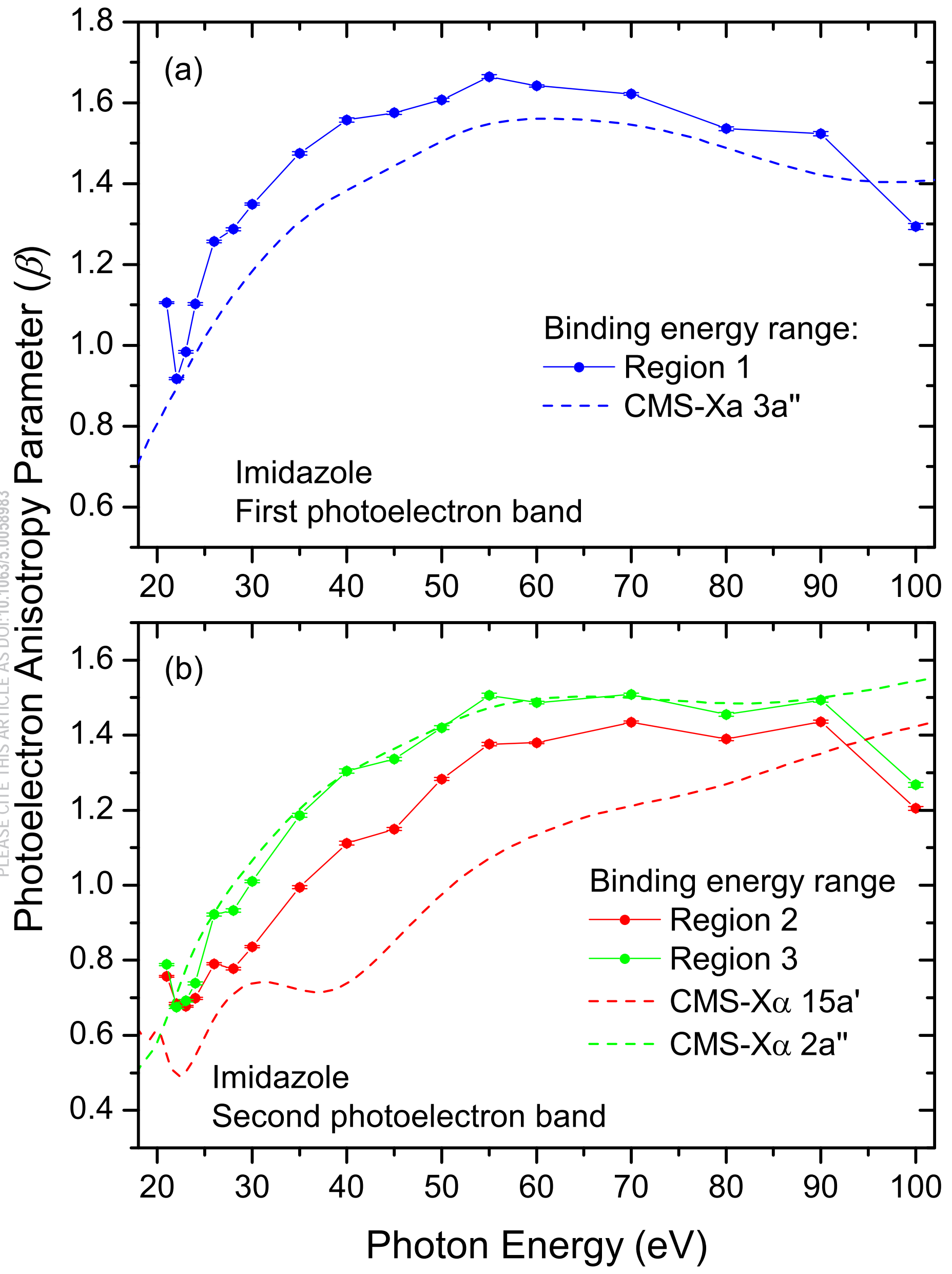




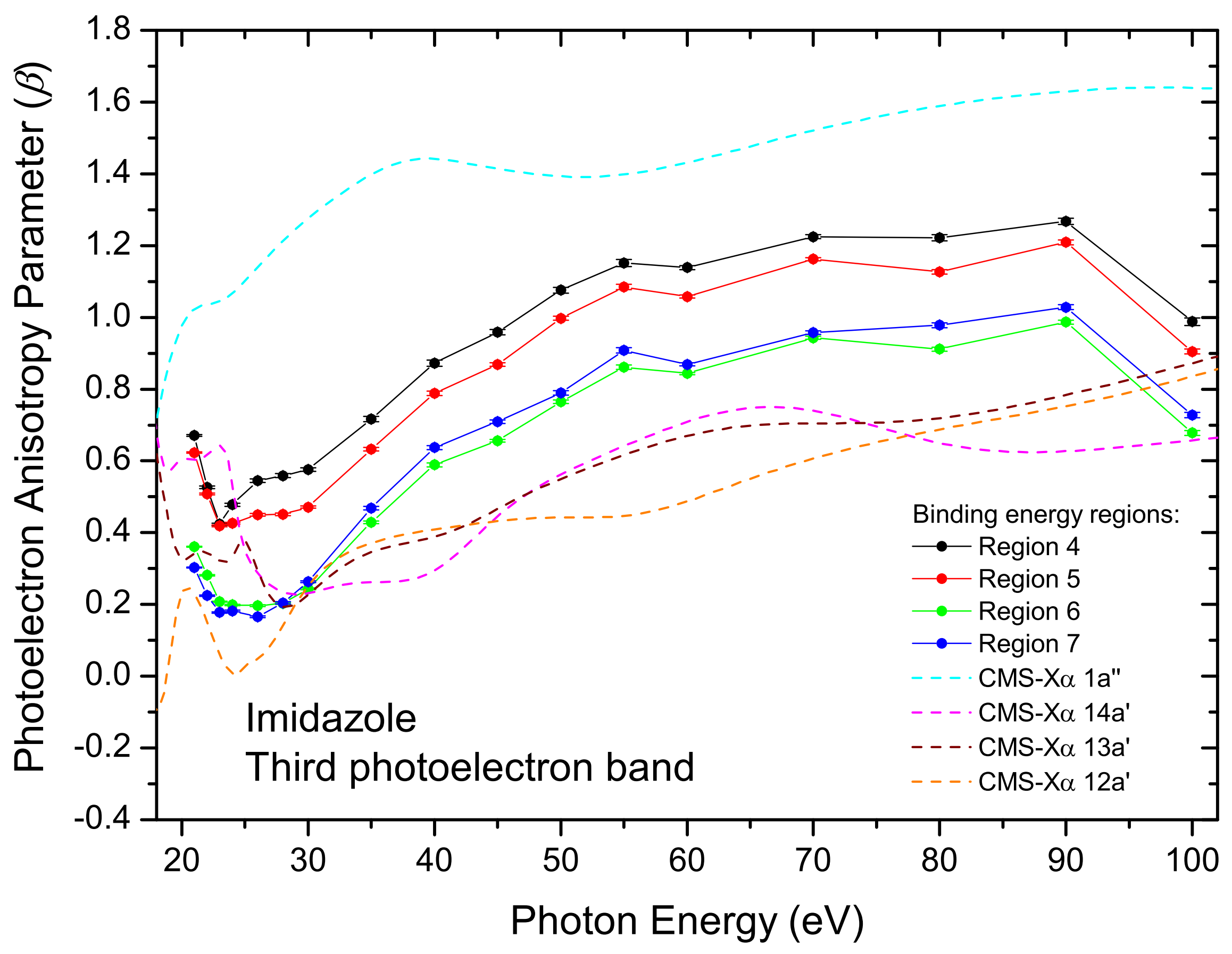




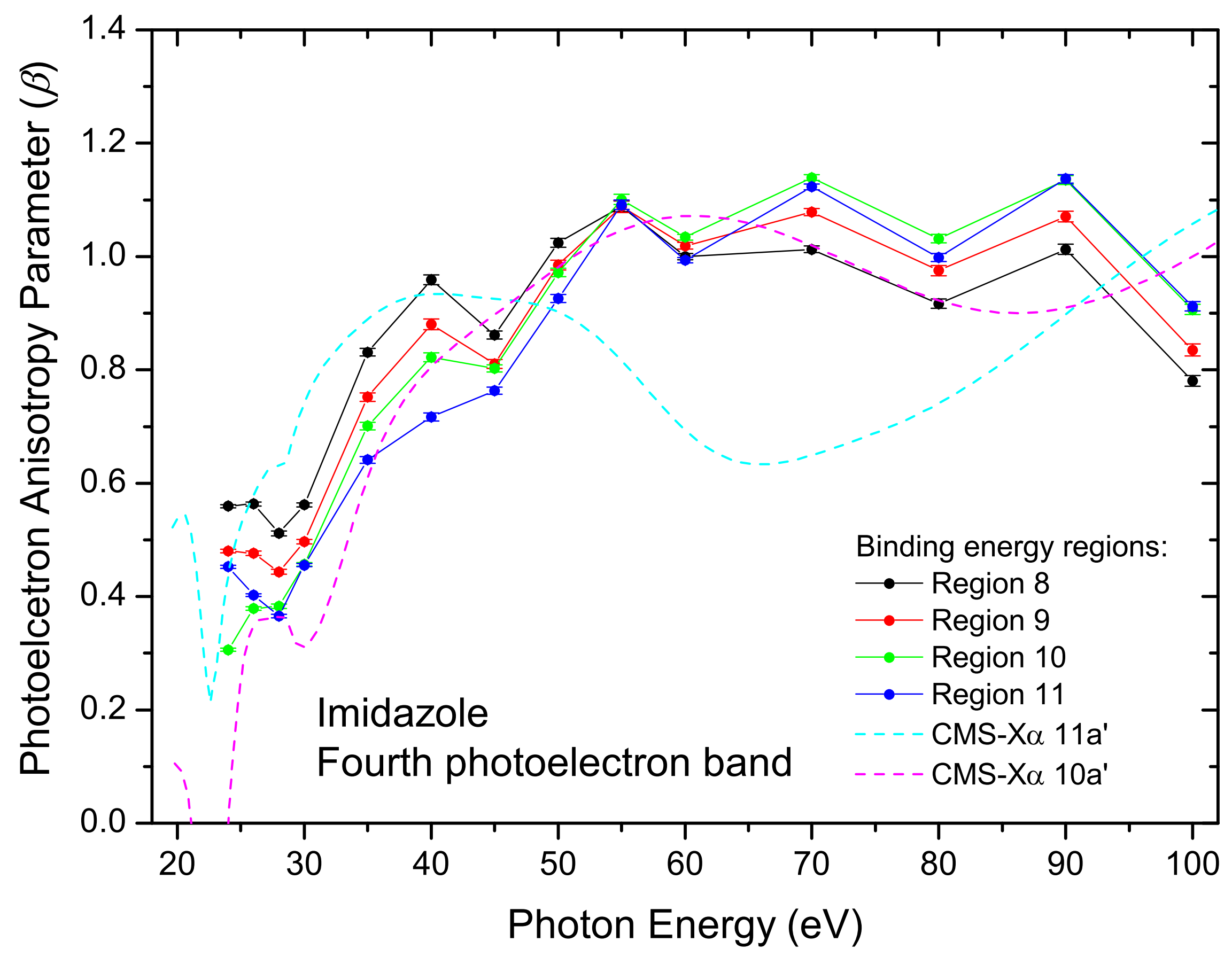

